Ján Filo · Stephan Luckhaus

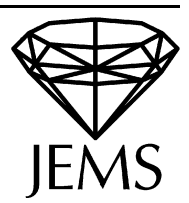

\title{
Homogenization of a boundary condition for the heat equation
}

Received August 20, 1999 / final version received March 1, 2000

Published online June 21, 2000 - (c) Springer-Verlag \& EMS 2000

\begin{abstract}
An asymptotic analysis is given for the heat equation with mixed boundary conditions rapidly oscillating between Dirichlet and Neumann type. We try to present a general framework where deterministic homogenization methods can be applied to calculate the second term in the asymptotic expansion with respect to the small parameter characterizing the oscillations.
\end{abstract}

\section{Introduction}

The paper deals with the asymptotic behaviour of solutions $u_{\varepsilon}$ of a homogenization problem for the heat equation in a cylindrical domain $\Omega \times(0, T)$ subject to mixed boundary conditions. The boundary conditions are rapidly oscilating between Dirichlet and Neumann type. Since the area fraction of the set $\mathcal{D}^{\varepsilon}$ where the homogeneous Dirichlet boundary conditions are prescribed is order 1 and in a sense "dense", in the first term of the expansion the Neumann boundary conditions are forgotten. The Neumann boundary conditions are prescribed on the set $\mathcal{N}^{\varepsilon}$, the complement of $\mathcal{D}^{\varepsilon}$ on $\partial \Omega$. In order to see the asymptotic effect of the Neumann boundary conditions on the solution one has to go to the second term of the expansion. In two dimensions and in a special geometric setting this problem was already considered in [6].

Here we try to present a "most general" framework where deterministic homogenization methods can still be applied to calculate the second term in the asymptotic expansion.

The condition we arrive at is roughly speaking asymptotic almost periodicity and as a special example quasi-periodicity of the characteristic function of the set $\mathcal{D}^{\varepsilon}$. Almost periodicity has to be understood in the metric

J. Filo: Institute of Applied Mathematics, Comenius University, Mlynská dolina, 84248 Bratislava, Slovakia

Supported by the Alexander von Humboldt Foundation

S. Luckhaus: Institut für Mathematik der Universität Leipzig, Augustusplatz 10/11, 04109 Leipzig, Germany

Mathematics Subject Classification (1991): 35K20, 35C20, 35B27 
given by $L^{2}$-capacity (see Assumption (P)). The homogenization method we employ is a blow-up technique combined with a suitably strong uniqueness result for solutions - in this case of a boundary problem for the Laplace equation in the half space. The technique is similar to the one used for convergence of saturation in the matrix in [3]. For other approaches for homogenization of boundary conditions see e.g. [8] and the references therein.

Our original aim was to study a flow problem in porous media with a part of the boundary covered by a fluid. For one incompressible fluid in a porous medium one has to solve the equation

$$
\frac{\partial \theta(p)}{\partial t}=\nabla \cdot(k(\theta(p))(\nabla p+e))
$$

where $p$ is the unknown pressure, $\theta$ the water content, $k$ the conductivity of the porous medium and $-e$ the direction of gravity (see [1] and references therein, for example). The part of the boundary which is covered by the fluid and where the infiltration takes place is supposed to behave like an impervious layer with many small holes. It is assumed that the holes are distributed "uniformly" and create a "periodic" structure with the period $\varepsilon$. The pressure is supposed to be 0 on the holes, where the fluid infiltrates into the porous medium and the zero flux condition $(k(\nabla p+e)) \cdot v=0$ is prescribed on the impervious part of the boundary. As the period and the diameter of the holes is of order $\varepsilon$ and the domain occupied by the porous medium is "large", it is natural to let

$$
\varepsilon \longrightarrow 0
$$

and to investigate the asymptotic behaviour of solutions to (1.1). Since this nonlinear problem was not yet treatable, here the linear heat equation is studied, i.e. $\theta(p) \equiv p$ and $k(\theta) \equiv 1$.

Our study initially, in Sect. 2, parallels the corresponding investigation in [6] concerning basic a priori estimates. It is demonstrated, besides others, that

$$
\left\|u_{\varepsilon}-u\right\|_{L^{2}(\Omega \times(0, T))}+\left\|u_{\varepsilon}-u\right\|_{L^{2}(\partial \Omega \times(0, T))} \leq C \varepsilon,
$$

where $u$ is the solution of the homogenized problem with purely Dirichlet boundary conditions. Section 3 investigates harmonic functions on the halfspace subject to mixed boundary conditions on the boundary hyperplane. Here the main result says that if a solution with homogeneous boundary conditions is sublinear, then it is the trivial solution. Using the blow-up argument, Sects. 4,5 provide a detailed analysis of a weak limit of the rescaled difference of the solution $u_{\varepsilon}$ and its first term $u$ in the expansion divided by $\varepsilon$. In Sect. 6 the limit $u^{1}$ of $\left(u_{\varepsilon}-u\right) / \varepsilon$ on $\Omega \times(0, T)$ is characterized as the solution of a first order homogenized problem, provided that some 
averages of limit solutions of scaled problems exist. This condition is the key subject of the paper and it is further analyzed in the concluding sections. In Sect. 7 a connection between a capacity assumption on a limit boundary set for Dirichlet data and an almost-periodicity condition which implies the requirement mentioned above is drawn. Finally, Sect. 8 presents an example of a nontrivial structure of the boundary set.

We finish this introductory section by introducing some notation. For a typical point in $\mathbb{R}^{n}$ we write $x=\left(x_{1}, \ldots, x_{n}\right)$ or $x=\left(x^{\prime}, x_{n}\right)$, where $x^{\prime}=\left(x_{1}, \ldots, x_{n-1}\right)$ and we set $\mathbb{R}_{+}^{n} \equiv\left\{x \in \mathbb{R}^{n} \mid x_{n}>0\right\}, \Omega_{T} \equiv \Omega \times(0, T)$, $\mathcal{N}_{T} \equiv \mathcal{N} \times(0, T)$, etc. An open ball in $\mathbb{R}^{n}$ with center $x$ and radius $r$ will be denoted by

$$
B(x, r) \equiv\left\{y \in \mathbb{R}^{n}|| x-y \mid<r\right\} \text { and } B(r) \equiv B(0, r) .
$$

Similarly in $\mathbb{R}^{n-1}$, i.e.

$$
B\left(x^{\prime}, r\right) \equiv\left\{y^{\prime} \in \mathbb{R}^{n-1}|| x^{\prime}-y^{\prime} \mid<r\right\} \quad \text { and } \quad B^{\prime}(r) \equiv B\left(0^{\prime}, r\right)
$$

and we set $B_{+}(r) \equiv B(r) \cap \mathbb{R}_{+}^{n}$. An open cube in $\mathbb{R}^{n}$ with a center $x$ and the side $2 r$ we denote $C(x, r) \equiv\left\{y \in \mathbb{R}^{n}|| x_{i}-y_{i} \mid<r, i=1,2, \ldots, n\right\}$ and $C(r) \equiv C(0, r)$. Analogously, $C\left(x^{\prime}, r\right)$ and $C^{\prime}(r)$ in $\mathbb{R}^{n-1} . \bar{E}$ denotes the closure of $E$. Next, set $A \triangle B \equiv A \cup B \backslash A \cap B$ for $A, B \subset \mathbb{R}^{n-1}$. We denote the average of $f$ over the set $Q \subset \mathbb{R}^{n}$ and over its boundary by

$$
\begin{gathered}
f_{Q} f d x=\frac{1}{\mathcal{L}^{n}(Q)} \int_{Q} f(x) d x, \\
f_{\partial Q} f d H^{n-1}(x)=\frac{1}{\mathcal{H}^{n-1}(\partial Q)} \int_{\partial Q} f(x) d H^{n-1}(x),
\end{gathered}
$$

where $\mathcal{L}^{n}$ is the $n$ dimensional Lebesgue measure in $\mathbb{R}^{n}$ and $\mathcal{H}^{n-1}$ is the $n-1$ dimensional Hausdorf measure in $\mathbb{R}^{n} . \alpha(k)=\pi^{k / 2} / \Gamma(1+k / 2)$ denotes volume of the unit ball in $\mathbb{R}^{k}$. By $\varepsilon$ we shall denote small parameter finally tending to zero and by $\rightarrow$ weak convergence. The function spaces we use are rather familiar and we omit the definitions. Finally, by $J \Phi$ we shall denote Jacobian of $\Phi$.

\section{The first term in the $\epsilon$-expansion}

In this section we study the problem

$$
\begin{aligned}
\partial_{t} u_{\varepsilon}-\Delta u_{\varepsilon} & =f_{\varepsilon}(x, t) & & \text { in } \Omega_{T}, \\
\partial_{\nu} u_{\varepsilon}+\sigma_{\varepsilon} u_{\varepsilon} & =\vartheta_{\varepsilon}(x, t) & & \text { on } \mathcal{N}_{T}^{\varepsilon}, \\
u_{\varepsilon} & =0 & & \text { on } \mathcal{D}_{T}^{\varepsilon}, \\
u_{\varepsilon} & =u_{0}^{\varepsilon} & & \text { on } \Omega \times\{t=0\}
\end{aligned}
$$

subject to the following assumptions: 
(A) $\Omega \subset \mathbb{R}^{n}$ is open, bounded and connected set with Lipschitz boundary $\partial \Omega$.

(B) For any $0<\varepsilon<1$ let

$$
\partial \Omega \equiv \mathcal{D}^{\varepsilon} \cup \mathcal{N}^{\varepsilon},
$$

where $\mathcal{D}^{\varepsilon}$ is a relatively open portion of $\partial \Omega$ fulfilling the following structure condition: There exist two numbers

$$
0<\delta<\rho
$$

such that for any $x^{0} \in \partial \Omega$ there is $x \in \mathcal{D}^{\varepsilon}$ satisfying

$$
B(x, \varepsilon \delta) \cap \partial \Omega \subset B\left(x^{0}, \varepsilon \rho\right) \cap \mathcal{D}^{\varepsilon} .
$$

(C) $f_{\varepsilon}, f, f^{1} \in L^{2}\left(\Omega_{T}\right)$ and such that

$$
F_{\varepsilon} \equiv \frac{f_{\varepsilon}-f}{\varepsilon} \rightarrow f^{1} \quad \text { in } \quad L^{2}\left(\Omega_{T}\right) \quad \text { as } \quad \varepsilon \rightarrow 0 .
$$

(D) $\sigma_{\varepsilon}, \partial_{t} \sigma_{\varepsilon} \in L^{\infty}\left(\partial \Omega_{T}\right)$ and there exists a positive constant $C$ independent of $\varepsilon$ such that $\left\|\sigma_{\varepsilon}\right\|_{L^{\infty}\left(\partial \Omega_{T}\right)} \leq C$.

(E) $\vartheta_{\varepsilon}, \vartheta, \partial_{t} \vartheta_{\varepsilon} \in L^{2}\left(\partial \Omega_{T}\right)$ and such that

$$
\vartheta_{\varepsilon} \rightarrow \vartheta \quad \text { in } L^{2}\left(\partial \Omega_{T}\right) \text { as } \varepsilon \rightarrow 0 .
$$

(F) $u_{0}^{\varepsilon}, u_{0} \in W^{1,2}(\Omega), u_{0}=0$ on $\partial \Omega, u_{0}^{\varepsilon}=0$ on $\mathcal{D}^{\varepsilon}, u_{0}^{1} \in L^{2}(\Omega)$ and such that

$$
\mathcal{P}_{\varepsilon} \equiv \frac{u_{0}^{\varepsilon}-u_{0}}{\varepsilon} \rightarrow u_{0}^{1} \quad \text { in } \quad L^{2}(\Omega) \quad \text { as } \varepsilon \rightarrow 0 .
$$

Note that some of the above assumptions can be relaxed, but for simplicity we do not give the most general hypotheses.

Now we state the rate of convergence of solutions $u_{\varepsilon}$ of (2.1) to the solution $u$ of the following problem:

$$
\begin{aligned}
\partial_{t} u & =\Delta u+f(x, t) & & \text { in } \Omega_{T}, \\
u & =0 & & \text { on } \partial \Omega_{T}, \\
u & =u_{0} & & \text { on } \Omega \times\{t=0\} .
\end{aligned}
$$

Theorem 2.1. Assume that (A)-(F) are satisfied. Then there exists a positive constant $C$, independent of $\varepsilon$, such that

$$
\max _{0 \leq t \leq T} \int_{\Omega}\left|u_{\varepsilon}-u\right|^{2}(x, t) d x+\int_{\Omega_{T}}\left|\nabla\left(u_{\varepsilon}-u\right)\right|^{2}(x, t) d x d t \leq C \varepsilon,
$$




$$
\begin{gathered}
\int_{\partial \Omega_{T}}\left|u_{\varepsilon}-u\right|^{2}(x, t) d H^{n-1}(x) d t+\int_{\Omega_{T}}\left|u_{\varepsilon}-u\right|^{2}(x, t) d x d t \leq C \varepsilon^{2}, \\
\max _{0 \leq t \leq T} \int_{\Omega}\left|u_{\varepsilon}-u\right|^{2}(x, t) \phi(x) d x \\
+\int_{\Omega_{T}}\left|\nabla\left(u_{\varepsilon}-u\right)\right|^{2}(x, t) \phi(x) d x d t \leq C \varepsilon^{2}
\end{gathered}
$$

and

$$
\begin{aligned}
\text { ess } \sup _{0 \leq t \leq T} \int_{\Omega}\left|\nabla\left(u_{\varepsilon}-u\right)\right|^{2}(x, t) \phi^{3}(x) d x & \\
& +\int_{\Omega_{T}}\left|\partial_{t}\left(u_{\varepsilon}-u\right)\right|^{2}(x, t) \phi^{3}(x) d x d t \leq C \varepsilon^{2},
\end{aligned}
$$

where $\phi$ is the principal eigenfunction of the problem

$$
\begin{aligned}
\Delta \phi+\lambda \phi & =0 & & \text { in } \Omega, \\
\phi & =0 & & \text { on } \partial \Omega,
\end{aligned}
$$

with the corresponding principal eigenvalue $\lambda=\lambda_{1}>0$.

Proof. This is almost exactly like the proof of the corresponding assertion of [6]. Therefore we prove here only the following

Proposition 2.2. Assume (A), (B) are satisfied. Then there exists a positive constant $C$ such that

$$
\|u\|_{L^{2}(\partial \Omega)} \leq C \sqrt{\varepsilon}\|u\|_{W^{1 / 2,2}(\partial \Omega)}
$$

for any $u \in W^{1,2}(\Omega), u=0$ on $\mathcal{D}^{\varepsilon}$.

Proof of Proposition 2.2. 1. Since $\partial \Omega$ is compact, it can be covered by a finite number of open sets $U^{1}, \ldots, U^{m}$ such that $\partial \Omega \cap U^{\ell}$ is the graph of a Lipschitz function and $\Omega \cap U^{\ell}$ is on one side of $\partial \Omega \cap U^{\ell}$. To be more precise, for each $\ell \in\{1, \ldots, m\}$ there exist an Euclidean coordinate system $e_{1}^{\ell}, \ldots, e_{n}^{\ell}$ in $\mathbb{R}^{n}$, positive numbers $r^{\ell}, h^{\ell}$ and a Lipschitz continuous function $\gamma^{\ell}: \mathbb{R}^{n-1} \rightarrow \mathbb{R}, \gamma^{\ell}(0)=0$, such that if we denote $x=\sum_{i=1}^{n} x_{i}^{\ell} e_{i}^{\ell}$ for $x \in \mathbb{R}^{n}$,

$$
\begin{gathered}
U^{\ell} \equiv\left\{x \in \mathbb{R}^{n}|| x_{i}^{\ell} \mid<r^{\ell} \text { and }\left|x_{n}^{\ell}-\gamma^{\ell}\left(x_{1}^{\ell}, \ldots, x_{n-1}^{\ell}\right)\right|<h^{\ell}\right\}, \\
\partial \Omega \cap U^{\ell} \equiv\left\{x \in \bar{\Omega} \mid x_{n}^{\ell}=\gamma^{\ell}\left(x_{1}^{\ell}, \ldots, x_{n-1}^{\ell}\right)\right\}
\end{gathered}
$$

and if $0<x_{n}^{\ell}-\gamma^{\ell}\left(x_{1}^{\ell}, \ldots, x_{n-1}^{\ell}\right)<h^{\ell}$, then $x \in \Omega$. Note next that

$$
\int_{\partial \Omega}|u(x)|^{2} d H^{n-1}(x) \leq \sum_{\ell=1}^{m} \int_{\partial \Omega \cap U^{\ell}}|u(x)|^{2} d H^{n-1}(x) .
$$


2. Fix now $\ell \in\{1, \ldots, m\}$ and denote

$$
v\left(y^{\prime}\right) \equiv u\left(\sum_{i=1}^{n-1} y_{i} e_{i}^{\ell}+\gamma^{\ell}\left(y^{\prime}\right) e_{n}^{\ell}\right), y^{\prime}=\left(y_{1}, \ldots, y_{n-1}\right) .
$$

Then

$$
\int_{\partial \Omega \cap U^{\ell}}|u(x)|^{2} d H^{n-1}(x)=\int_{C^{\prime}(r)}\left|v\left(y^{\prime}\right)\right|^{2} a\left(y^{\prime}\right) d y^{\prime},
$$

where $C^{\prime}(r) \equiv\left\{y^{\prime} \in \mathbb{R}^{n-1}|| y_{i} \mid<r, i=1, \ldots, n-1\right\}$ and

$$
a\left(y^{\prime}\right)=\left(1+\left|D \gamma\left(y^{\prime}\right)\right|^{2}\right)^{1 / 2} .
$$

For simplicity of notation we shall not indicate the dependence of the data on $\ell$.

3. Take $C^{\prime}(r)$ and divide it into $k_{\varepsilon}^{n-1}$ subcubes of side length $r / k_{\varepsilon}$, where

$$
k_{\varepsilon}=\left[\frac{r}{\varepsilon \rho}\right] \text {. }
$$

Call these subcubes $Q_{1}, \ldots, Q_{k_{\varepsilon}^{n-1}}$. Then

$$
\int_{\partial \Omega \cap U^{\ell}}|u(x)|^{2} d H^{n-1}(x)=\sum_{j=1}^{k_{\varepsilon}^{n-1}} \int_{Q_{j}}\left|v\left(y^{\prime}\right)\right|^{2} a\left(y^{\prime}\right) d y^{\prime} .
$$

Observe now

$$
\int_{Q_{j}}\left|v\left(y^{\prime}\right)\right|^{2} a\left(y^{\prime}\right) d y^{\prime}=\int_{Q_{j}} \frac{\left|v\left(y^{\prime}\right)-v\left(z^{\prime}\right)\right|^{2}}{\left|y^{\prime}-z^{\prime}\right|^{n}}\left|y^{\prime}-z^{\prime}\right|^{n} a\left(y^{\prime}\right) d y^{\prime}
$$

for any $z^{\prime} \in D_{j}^{\varepsilon}$, where

$$
D_{j}^{\varepsilon} \equiv\left\{y^{\prime} \in Q_{j} \mid x=\sum_{i=1}^{n-1} y_{i} e_{i}^{\ell}+\gamma^{\ell}\left(y^{\prime}\right) e_{n}^{\ell} \in \mathcal{D}^{\varepsilon}\right\} .
$$

We claim that there exists a positive constant $K$, independent of $j$ and $\varepsilon$, such that

$$
\mathcal{L}^{n-1}\left(D_{j}^{\varepsilon}\right) \geq K(\delta \varepsilon)^{n-1} .
$$

Indeed, (B) yields the the existence $x^{j} \in \mathcal{D}^{\varepsilon}$ such that

$$
\begin{aligned}
B\left(x^{j}, \varepsilon \delta\right) \cap \partial \Omega & \subset \mathcal{D}_{j}^{\varepsilon} \\
& \equiv\left\{x \in \mathcal{D}^{\varepsilon} \mid x=\sum_{i=1}^{n-1} y_{i} e_{i}^{\ell}+\gamma^{\ell}\left(y^{\prime}\right) e_{n}^{\ell}, y^{\prime} \in D_{j}^{\varepsilon}\right\} .
\end{aligned}
$$


Then, however,

$$
\begin{aligned}
\mathcal{L}^{n-1} & \left(B^{\prime}\left(\varepsilon \delta / \sqrt{1+\operatorname{Lip}^{2}(\gamma)}\right)\right) \\
& \leq \mathcal{H}^{n-1}\left(\mathcal{D}_{j}^{\varepsilon}\right)=\int_{D_{j}^{\varepsilon}} a\left(y^{\prime}\right) d y^{\prime} \leq C_{1} \mathcal{L}^{n-1}\left(D_{j}^{\varepsilon}\right)
\end{aligned}
$$

and the estimate (2.6) follows. Note that by $\operatorname{Lip}(\gamma)$ we mean Lipschitz constant of $\gamma$.

4. Integrate now (2.5) over $D_{j}^{\varepsilon}$ through $z^{\prime}$ and one easily arrives at

$$
\begin{aligned}
& \int_{Q_{j}}\left|v\left(y^{\prime}\right)\right|^{2} a\left(y^{\prime}\right) d y^{\prime} \\
& \quad \leq \varepsilon \frac{C_{2} \rho^{n-1}}{\delta^{n-1}} \int_{D_{j}^{\varepsilon}} \int_{Q_{j}} \frac{\left|v\left(y^{\prime}\right)-v\left(z^{\prime}\right)\right|^{2}}{\left|y^{\prime}-z^{\prime}\right|^{n}} a\left(y^{\prime}\right) a\left(z^{\prime}\right) d y^{\prime} d z^{\prime} .
\end{aligned}
$$

Summing on $j$ gives

$$
\begin{aligned}
\int_{\partial \Omega \cap U^{\ell}} & |u(x)|^{2} d H^{n-1}(x) \\
\leq & C_{3} \varepsilon \int_{\partial \Omega \cap U^{\ell}} \int_{\partial \Omega \cap U^{\ell}} \frac{|u(y)-u(z)|^{2}}{|y-z|^{n}} d H^{n-1}(y) d H^{n-1}(z),
\end{aligned}
$$

and finally, adding up through $\ell=1, \ldots, m$ we arrive at (2.4).

\section{Harmonic functions on the half-space}

This section studies harmonic functions in the half space subject to the mixed boundary condition. We will here mostly deal with the boundaryvalue problem

$$
\begin{array}{rlrl}
\Delta \omega & =0 & & \text { in } \mathbb{R}_{+}^{n}, \\
\omega=0 & & \text { on } \mathbb{D}_{0} \equiv \mathbb{D} \times\left\{x_{n}=0\right\}, \\
\partial_{\nu} \omega=g & & \text { on } \mathbb{N}_{0} \equiv \mathbb{N} \times\left\{x_{n}=0\right\},
\end{array}
$$

where $g \in \mathbb{R}$ is given, $\partial_{\nu} \omega=-\partial \omega / \partial x_{n}, \mathbb{N}=\mathbb{R}^{n-1} \backslash \mathbb{D}$ and an open set $\mathbb{D} \subset \mathbb{R}^{n-1}$ is asymptotically dense in $\mathbb{R}^{n-1}$ in the sense of the following definition.

Definition 3.1. A set $\mathbb{D} \subset \mathbb{R}^{n-1}$ is said to be asymptotically dense in $\mathbb{R}^{n-1}$ if there exist positive numbers $\delta, \rho$ such that any ball with radius $\rho$ in $\mathbb{R}^{n-1}$ contains at least one ball with radius $\delta$ entirely lying in $\mathbb{D}$, i.e. for any $y^{\prime} \in \mathbb{R}^{n-1}$ there exists at least one point $x^{\prime} \in B\left(y^{\prime}, \rho\right)$ such that

$$
B\left(x^{\prime}, \delta\right) \subset B\left(y^{\prime}, \rho\right) \cap \mathbb{D} .
$$


Definition 3.2. We say $\omega \in W_{\text {loc }}^{1,2}\left(\mathbb{R}_{+}^{n}\right)$ is a weak solution of Problem (3.1) if $\omega=0$ on $\mathbb{D}_{0}$ and the integral identity

$$
\int_{\mathbb{R}_{+}^{n}} \nabla \omega \nabla \psi d x=\int_{\mathbb{R}^{n-1}} g \psi\left(x^{\prime}, 0\right) d x^{\prime}
$$

holds for any $\psi \in W_{\text {loc }}^{1,2}\left(\mathbb{R}_{+}^{n}\right), \psi=0$ on $\mathbb{D}_{0}$, with a compact support in $\overline{\mathbb{R}}_{+}^{n}$.

Note

$$
\omega=-g x_{n}
$$

is a solution of Problem (3.1). We will see in a moment, that there is also a bounded solution of our problem. Thus, the difference of those solutions satisfies

$$
\begin{aligned}
\Delta \omega=0 & \text { in } \mathbb{R}_{+}^{n}, \\
\omega=0 & \text { on } \mathbb{D}_{0}, \\
\partial_{\nu} \omega=0 & \text { on } \mathbb{N}_{0},
\end{aligned}
$$

and does not equal zero. The main result of this section says, however, that if a solution of (3.4) is sublinear, then it is the trivial solution.

Theorem 3.3. Assume $\omega$ is a solution of Problem (3.4) in the sense of Definition 3.2. Suppose further $\omega$ satisfies

$$
\liminf _{R \rightarrow \infty} f_{B_{+}(R)}|\omega| d x / R=0 .
$$

Then

$$
\omega \equiv 0 .
$$

Proof. 1. First of all, if we define

$$
u(x) \equiv\left\{\begin{array}{ll}
\omega(x) & \text { if } x_{n} \geq 0 \\
\omega\left(x^{\prime},-x_{n}\right) & \text { if } x_{n}<0
\end{array},\right.
$$

then $|u(x)|$ is a weak subharmonic function, i.e. $|u| \in W_{l o c}^{1,2}\left(\mathbb{R}^{n}\right)$ and

$$
\int_{\mathbb{R}_{+}^{n}} \nabla|u| \nabla \psi d x \leq 0
$$

for any $\psi \in W_{l o c}^{1,2}\left(\mathbb{R}^{n}\right), \psi \geq 0$, with a compact support. Therefore,

$$
|u(x)| \leq f_{B(x, R)}|u(y)| d y
$$


and thus,

$$
\sup _{B_{+}(R)}|\omega| \leq 2^{n} f_{B_{+}(2 R)}|\omega| d x
$$

Now let $\left\{R_{i}\right\}_{i=1}^{\infty}, R_{i} \rightarrow \infty$ as $i \rightarrow \infty$ be such that

$$
\lim _{i \rightarrow \infty} f_{B_{+}\left(2 R_{i}\right)}|\omega| d x / R_{i}=0 .
$$

Our intention next is to deal with the problem in the half ball

$$
\begin{aligned}
\Delta \omega & =0 & & \text { in } B_{+}(R), \\
\omega & =0 & & \text { on } \mathbb{D}_{0} \cap B(R), \\
\partial_{\nu} \omega & =0 & & \text { on } \mathbb{N}_{0} \cap B(R),
\end{aligned}
$$

assuming that

$$
\sup _{B_{+}(R)}|\omega|=M
$$

2. Claim \#1: There exist constants $0<\gamma<1,0<d<\infty$ such that

$$
\sup _{B^{\prime}\left(R-4 k-\sum_{j=1}^{k} d_{j}\right) \times(0,1)}|\omega| \leq \gamma^{k} M
$$

for

$$
d_{j} \equiv \frac{d}{\gamma^{j}}
$$

$j \in\{1, \ldots, k\}$, whenever $4 k+\sum_{j=1}^{k} d_{j}<R$. The constants $\gamma, d$ are independent of $R, M$.

Before proving Claim \#1, let us introduce two auxiliary assertions.

3. Claim \#2: Assume

$$
|\omega| \leq K
$$

in $B^{\prime}(L) \times(0,4), 4<L \leq R$. Then

$$
|\omega| \leq \beta K
$$

in $B^{\prime}(L-4) \times(0,1)$ for some $0<\beta<1$, independent of $R, K$ and $L$.

Note that for simplicity of notation we have put $\rho=1$ in Definition 3.1 which applies here. 
Proof of Claim \#2. It suffices to prove the following statement. Let $z=$ $\left(z^{\prime}, 0\right)$ be such that $B(z, 4) \subset B(R)$ and

$$
\sup _{B_{+}(z, 4)}|\omega| \leq K .
$$

Then

$$
\sup _{B_{+}(z, 1)}|\omega| \leq \beta K
$$

for some $0<\beta<1$.

As $\mathbb{D}$ is asymptotically dense, there is $y=\left(y^{\prime}, 0\right) \in \mathbb{D}_{0}$ such that $B\left(y^{\prime}, \delta\right) \subset \mathbb{D}$ and $B(y, 3) \subset B(R)$. Moreover

$$
\begin{aligned}
\Delta \omega=0 & & \text { in } B_{+}(y, 3), \\
\omega=0 & & \text { on } \mathbb{D}_{0} \cap B(y, 3), \\
\partial_{\nu} \omega=0 & & \text { on } \mathbb{N}_{0} \cap B(y, 3)
\end{aligned}
$$

and

$$
\sup _{B_{+}(y, 3)}|\omega| \leq K .
$$

If we compare $\omega$ with the solutions $v_{+}, v_{-}$of the problem

$$
\begin{aligned}
\Delta v & =0 & & \text { in } B_{+}(y, 3), \\
v & =0 & & \text { on } B\left(y^{\prime}, \delta\right) \times\left\{x_{n}=0\right\}, \\
\partial_{\nu} v & =0 & & \text { on }\left(B\left(y^{\prime}, 3\right) \backslash B\left(y^{\prime}, \delta\right)\right) \times\left\{x_{n}=0\right\}, \\
v & = \pm K & & \text { on } \partial B_{+}(y, 3) \cap \mathbb{R}_{+}^{n},
\end{aligned}
$$

we obtain

$$
-\beta K \leq v_{-} \leq \omega \leq v_{+} \leq \beta K
$$

on $B_{+}(y, 2)$ for some $0<\beta<1$, i.e. (3.10) holds and the assertion of Claim \#2 follows easily.

4. Claim \#3: Let $\omega, R, M$ be as above, $|\omega| \leq M$ on $B_{+}(R)$ and let $0<\varepsilon<1$, $0<c<\infty$ and $0<\alpha<1$ be given. Moreover, choose a constant $\ell>0$ such that

$$
\frac{(1-\alpha) 4 c}{\ell} \leq \varepsilon .
$$

Then

$$
|\omega| \leq(\alpha+\varepsilon) M
$$

on $B^{\prime}(L-\ell) \times(0,4)$, whenever

$$
|\omega| \leq \alpha M
$$

on $B^{\prime}(L) \times\left\{x_{n}=0\right\}$. 
Proof of Claim \#3. Let $z=\left(z^{\prime}, 0\right)$ and $\varrho>0$. Consider first the problem

$$
\begin{aligned}
\Delta u_{\varrho}=0 & \text { in } B_{+}(z, 2 \varrho), \\
u_{\varrho}=0 & \text { on } B\left(z^{\prime}, 2 \varrho\right) \times\left\{x_{n}=0\right\}, \\
u_{\varrho}=1 & \text { on } \partial B_{+}(z, 2 \varrho) \cap \mathbb{R}_{+}^{n} .
\end{aligned}
$$

Then there exist positive constants $c_{1}, c_{2}$ (independent of $\varrho$ ) such that

$$
\frac{c_{1}}{\varrho} \leq \frac{\partial u_{\varrho}}{\partial x_{n}} \leq \frac{c_{2}}{\varrho}
$$

for any $x \in B_{+}(z, \varrho)$. This follows easily, if we first consider $\varrho=1$ and then put $u_{\varrho}(x) \equiv u_{1}(x / \varrho)$.

Assume now that $|\omega| \leq M$ on $B_{+}(R),|\omega| \leq \alpha M$ on $B^{\prime}(L) \times\left\{x_{n}=0\right\}$ and $\ell$ is choosen in such a way that

$$
\frac{(1-\alpha) c_{2} 4}{\ell} \leq \varepsilon
$$

for given $\varepsilon$. Let $z$ in (3.12) be such that

$$
|z| \leq L-2 \ell .
$$

Then it is not difficult to see that

$$
|\omega| \leq \alpha M+(1-\alpha) M u_{\ell}
$$

in $B_{+}(z, 2 \ell)$. Hence,

$$
|\omega| \leq \alpha M+(1-\alpha) M 4 c_{2} / \ell
$$

on $B^{+}(z, \ell) \cap\left\{x \mid x_{n} \leq 4\right\}$, i.e. $|\omega| \leq(\alpha+\varepsilon) M$. As this holds for any $z=\left(z^{\prime}, 0\right),|z| \leq R-2 \ell,(3.11)$ holds on $B^{\prime}(R-\ell) \times(0,4)$. Claim \#3 is proved.

5. Proof of Claim \#1. According to Claim \#2,

$$
\sup _{B^{\prime}(R-4) \times(0,1)}|\omega| \leq \beta M
$$

for given $0<\beta<1$. Put $c=c_{2}$ (see (3.13)) in Claim \#3 and we have

$$
\sup _{B^{\prime}(R-4-\ell) \times(0,4)}|\omega| \leq\left(\beta+\varepsilon_{1}\right) M,
$$

where $\varepsilon_{1} \ell=4 c$. Choose $\varepsilon_{0}>0$ such small that

$$
\gamma \equiv \beta\left(1+\varepsilon_{0}\right)<1
$$


and set $\varepsilon_{1}=\beta \varepsilon_{0}$. Then

$$
\sup _{B^{\prime}\left(R-4-d_{1}\right) \times(0,4)}|\omega| \leq \gamma^{1} M,
$$

for

$$
d_{1}=\frac{4 c}{\beta \varepsilon_{0}}=\frac{4 c\left(1+\varepsilon_{0}\right)}{\varepsilon_{0}} \frac{1}{\gamma^{1}} .
$$

Now we apply Claim \#2 again, and we arrive at

$$
\sup _{B^{\prime}\left(R-8-d_{1}\right) \times(0,1)}|\omega| \leq \beta \gamma M,
$$

that due to Claim \#3 gives

$$
|\omega| \leq\left(\beta \gamma+\varepsilon_{2}\right) M
$$

on $B^{\prime}\left(R-8-d_{1}-d_{2}\right) \times(0,4)$. Taking $\varepsilon_{2}=\gamma \beta \varepsilon_{0}$,

$$
|\omega| \leq \beta \gamma\left(1+\varepsilon_{0}\right) M=\gamma^{2} M
$$

and

$$
d_{2}=\frac{4 c}{\beta \varepsilon_{0} \gamma}=\frac{4 c\left(1+\varepsilon_{0}\right)}{\varepsilon_{0}} \frac{1}{\gamma^{2}} .
$$

Hence, by iteration we get the required estimate (3.9) for

$$
d_{j} \equiv \frac{d}{\gamma^{j}}, \quad d=\frac{4 c\left(1+\varepsilon_{0}\right)}{\varepsilon_{0}} .
$$

This proves Claim \#1.

6. Finally, we are now prepared to prove Theorem 3.3.

Choose first integers $k_{i}$ such that

$$
\nu R_{i} \leq 4 k_{i}+\sum_{j=1}^{k_{i}} d_{j} \leq R_{i} / 2,
$$

where $R_{i}$ are given by (3.7). It is not difficult to see that this is possible if $v$ is sufficiently small, say $2 v \leq \gamma$. Moreover, note that $k_{i} \leq \kappa \ln R_{i}$ for some positive constant $\kappa$. Next, Claim \#1 yields

$$
\sup _{B^{\prime}\left(R_{i} / 2\right) \times(0,1)}|\omega| \leq \frac{\sup _{B_{+}\left(R_{i}\right)}|\omega|}{v R_{i}-4 k_{i}+\text { const. }}
$$

that together with (3.6) yields

$$
\sup _{B^{\prime}\left(R_{i} / 2\right) \times(0,1)}|\omega| \leq C f_{B_{+}\left(2 R_{i}\right)}|\omega| d x / R_{i}
$$

for some positive constant $C$ independent of $i$. Making use of (3.7) one easily gets $\omega \equiv 0$ on $\mathbb{R}_{+}^{n}$. 
Theorem 3.4 (Existence of weak solution). There exists a bounded weak solution of (3.1). Moreover, this solution is positive in $\mathbb{R}_{+}^{n}$ if $g>0$ and $\mathbb{N}_{0} \neq \emptyset$.

Proof. The bounded solution of Problem (3.1) arises as the limit of weak solutions to the problems on bounded domains:

$$
\begin{aligned}
\Delta u_{k}=0 & & \text { in } B_{+}(k), \\
u_{k}=0 & & \text { on } \mathbb{D}_{0} \cap B(k), \\
u_{k}=0 & & \text { on } S_{k}, \\
\partial_{\nu} u_{k}=1 & & \text { on } \mathbb{N}_{0} \cap B(k),
\end{aligned}
$$

as $k \longrightarrow \infty$, where $S_{k} \equiv\left\{x \in \bar{B}_{+}(k)|| x \mid=k\right.$ and $\left.x_{n}>0\right\}$.

Note, that without loss of generality we can consider $g=1$, as otherwise the result follows by multiplication.

Lemma 3.5. There exists a positive constant $M$, independent of $k$, such that

$$
0<u_{k} \leq M \quad \text { on } \quad B_{+}(k) .
$$

Proof of Lemma 3.5. 1. As $\mathbb{D}$ is supposed to be asymptotically dense in $\mathbb{R}^{n-1}$, the following statement follows.

Claim \#1: There exists a sequence $\left\{z_{i}^{\prime}\right\}_{i=1}^{\infty}, \quad z_{i}^{\prime} \in \mathbb{R}^{n-1}$ such that $B\left(z_{i}^{\prime}, \delta\right) \subset \mathbb{D}$,

$$
B\left(z_{i}^{\prime}, 4 \rho\right) \cap B\left(z_{j}^{\prime}, 4 \rho\right)=\emptyset(i \neq j) \text { and } \mathbb{R}^{n-1} \subset \bigcup_{i=1}^{\infty} C\left(z_{i}^{\prime}, 10 \rho\right) \text {. }
$$

2. Next, let $v$ be a bounded nonnegative solution of the problem

$$
\begin{aligned}
\Delta v & =0 & & \text { in } \mathbb{R}_{+}^{n}, \\
v & =f & & \text { on } \mathbb{R}^{n-1} \times\left\{x_{n}=0\right\},
\end{aligned}
$$

where $f \in C^{\infty}\left(\mathbb{R}^{n-1}\right), 0 \leq f \leq 1$ and

$$
f\left(x^{\prime}\right)= \begin{cases}0 & \text { if } x^{\prime} \in \bigcup_{i=1}^{\infty} B\left(z_{i}^{\prime}, \delta / 2\right), \\ 1 & \text { if } x^{\prime} \in \mathbb{R}^{n-1} \backslash \bigcup_{i=1}^{\infty} B\left(z_{i}^{\prime}, \delta\right) .\end{cases}
$$

We claim that there exists a constant $0<\beta<1$ such that

$$
0<v(x) \leq 1-\beta \quad \text { on } \bigcup_{i=1}^{\infty} B\left(z_{i}, 2 \rho\right),
$$

where

$$
z_{i}=\left(z_{i}^{\prime}, 4 \rho\right)
$$


To see this, suppose the contrary and denote by $\left\{z_{k}^{\prime}\right\}_{k=1}^{\infty}$ the sequence of those points of $\left\{z_{i}^{\prime}\right\}_{i=1}^{\infty}$ for which

$$
\max _{\bar{B}\left(z_{k}, 2 r\right)} v(x) \longrightarrow 1 \quad \text { as } k \rightarrow \infty
$$

$z_{k}=\left(z_{k}^{\prime}, 4 \rho\right)$. Write

$$
Q \equiv B^{\prime}(4 \rho) \times(0,6 \rho)
$$

and define

$$
w_{k}(x) \equiv v\left(x^{\prime}+z_{k}^{\prime}, x_{n}\right)
$$

for $x \in Q$. So we get the sequence of harmonic functions in $Q$ such that

$$
\begin{gathered}
0 \leq w_{k} \leq 1 \quad \text { on } \quad \bar{Q}, \\
w_{k}\left(x_{k}\right) \longrightarrow 1 \quad \text { as } k \rightarrow \infty
\end{gathered}
$$

for $x_{k} \in \bar{B}(a, 2 \rho), a=\left(0^{\prime}, 2 \rho\right)$ and

$$
f_{\partial Q} w_{k}(x) d H^{n-1}(x) \leq 1-\alpha
$$

for some $0<\alpha<1$ and each $k$. Hence, there exists a subsequence $\left\{w_{k_{i}}\right\}_{i=1}^{\infty}$ converging uniformly on $\bar{B}(a, 2 \rho)$, to a harmonic function $w$ and $w\left(x_{\infty}\right)=1$, where $x_{k_{i}} \rightarrow x_{\infty}$ as $i \rightarrow \infty$. Then, however, $w \equiv 1$ on $Q-$ a contradiction to (3.19), as $w_{k_{i}} \rightarrow w$ weakly star in $L^{\infty}(\partial Q)$. Indeed, note that

$$
\int_{\partial Q} w_{k_{i}} \partial_{\nu} \psi=\int_{Q} w_{k_{i}} \Delta \psi
$$

for any sufficiently smooth test function $\psi$ such that $\psi=0$ on $\partial Q$. We can let $i \rightarrow \infty$ to obtain

$$
\int_{\partial Q} w \partial_{\nu} \psi=\int_{Q} \Delta \psi=\int_{\partial Q} \partial_{\nu} \psi .
$$

Assuming, that $\partial_{\nu} \psi \equiv 1$ on $\partial Q$ we obtain

$$
f_{\partial Q} w(x) d H^{n-1}(x)=1,
$$

that contradicts to (3.19), as we have mentioned above. 
3. Furthermore, let $i \in\{1,2, \ldots\}$ be arbitrary and denote by $v_{i}$ the bounded solution of the problem

$$
\begin{aligned}
& \Delta v_{i}=0 \quad \text { in } \quad \mathbb{R}_{+}^{n} \backslash B\left(z_{i}, 2 \rho\right), \\
& v_{i}=1-\beta \quad \text { on } \quad \partial B\left(z_{i}, 2 \rho\right) \text {, } \\
& v_{i}=1 \quad \text { on } \quad \mathbb{R}^{n-1} \times\left\{x_{n}=0\right\} \text {. }
\end{aligned}
$$

It follows from Hopf's lemma, that there exists a positive number $\vartheta$ such that

$$
\partial_{\nu} v_{i}\left(x^{\prime}, 0\right) \geq \vartheta>0
$$

for any $x^{\prime} \in B\left(z_{i}^{\prime}, 10 \rho\right)$. Owing to estimate (3.20) we prove

Claim \#2: $\partial_{\nu} v \geq \vartheta>0$ on $\mathbb{R}^{n-1} \backslash \bigcup_{i=1}^{\infty} B\left(z_{i}^{\prime}, \delta\right)$.

Proof of Claim \#2. Fix $i \in\{1,2, \ldots\}$. As

$$
v \leq v_{i} \quad \text { and } \quad v=v_{i}=1 \quad \text { on }\left(\mathbb{R}^{n-1} \backslash B\left(z_{i}^{\prime}, \delta\right)\right) \times\left\{x_{n}=0\right\},
$$

one easily gets

$$
0<\vartheta \leq \partial_{\nu} v_{i} \leq \partial_{\nu} v \quad \text { on } \quad\left(\mathbb{R}^{n-1} \backslash B\left(z_{i}^{\prime}, \delta\right)\right) \times\left\{x_{n}=0\right\} .
$$

As this holds for any $i$, the assertion of Claim \#2 follows.

4. Finally, set

$$
U=\frac{v}{\vartheta} .
$$

We utilize (3.17) and Claim \#2 to conclude that

$$
\begin{aligned}
\Delta U=0 & \text { in } \mathbb{R}_{+}^{n}, \\
U=0 & \text { on } \bigcup_{i=1}^{\infty} B\left(z_{i}^{\prime}, \delta / 2\right) \times\left\{x_{n}=0\right\}, \\
U \geq 0 & \text { on } \bigcup_{i=1}^{\infty}\left(B\left(z_{i}^{\prime}, \delta\right) \backslash B\left(z_{i}^{\prime}, \delta / 2\right)\right) \times\left\{x_{n}=0\right\}, \\
\partial_{\nu} U \geq 1 & \text { on }\left(\mathbb{R}^{n-1} \backslash \bigcup_{i=1}^{\infty} B\left(z_{i}^{\prime}, \delta\right)\right) \times\left\{x_{n}=0\right\} .
\end{aligned}
$$

Hence, 0 is a subsolution and $U$ is a supersolution to Problem (3.15) for any $k$, i.e.

$$
0 \leq u_{k} \leq U \quad \text { on } \quad B_{+}(k) .
$$

The fact, that $u_{k}>0$ on $B_{+}(k)$ follows from the fact that harmonic function $u_{k} \not \equiv 0$ cannot attain its minimum in interior point of $B_{+}(k)$. This proves Lemma 3.5. 
Finally, it follows from (3.16) that

$$
\int_{B_{+}(K)}\left|\nabla u_{k}\right|^{2} \leq M \mathcal{L}^{n-1}\left(B^{\prime}(K)\right)
$$

for any $k \geq K$. Hence, by the diagonal process we obtain a sequence $\left\{u_{k_{j}}\right\}_{j=1}^{\infty}$ such that it converges to a bounded weak solution $\omega$ of Problem (3.1) on any compact subset of $\overline{\mathbb{R}}_{+}^{n}$.

\section{Blow-up}

Assume for this and the next sections that $\Omega \subset \mathbb{R}^{n}$, that we have already supposed to be open, bounded and connected set, is $C^{2}$, i.e. near each point $x \in \partial \Omega, \partial \Omega$ is the graph of a $C^{2}$ function. For our purposes, however, it will be convenient to formulate it more precisely.

Definition 4.1. We say $\partial \Omega$ is $C^{2}$ if there is $r>0$ such that for each point $x^{0} \in \partial \Omega$, there exist an orthogonal matrix $\boldsymbol{O}$ and a $C^{2}$ mapping $\gamma: \mathbb{R}^{n-1} \rightarrow \mathbb{R}, \gamma(0)=0, D \gamma(0)=0$ such that we have

$$
\Omega \cap B\left(x^{0}, r\right)=\left\{x \in \mathbb{R}^{n} \mid x=x^{0}+\boldsymbol{O} \xi, \gamma\left(\xi^{\prime}\right)<\xi_{n}\right\} \cap B\left(x^{0}, r\right) .
$$

Note that $\boldsymbol{O}, \boldsymbol{\gamma}$ depend on $x^{0}$ and we write down this dependence as $\boldsymbol{O}\left(x^{0}\right)$ and $\gamma\left(x^{0}, \cdot\right)$, if it needs to be.

Notation. Fix $x^{0} \in \partial \Omega, 0<\varepsilon \ll 1$, and set

$$
\begin{gathered}
\Omega_{\varepsilon}\left(x^{0}\right) \equiv\left\{y \in \mathbb{R}^{n} \mid x=x^{0}+\varepsilon \boldsymbol{O} y \in \Omega\right\}, \\
\partial \Omega_{\varepsilon}\left(x^{0}\right) \equiv\left\{y \in \mathbb{R}^{n} \mid x=x^{0}+\varepsilon \boldsymbol{O} y \in \partial \Omega\right\}, \\
\mathcal{D}_{\varepsilon}\left(x^{0}\right) \equiv\left\{y \in \mathbb{R}^{n} \mid x=x^{0}+{ }_{\varepsilon} \boldsymbol{O} y \in \mathcal{D}^{\varepsilon} \subset \partial \Omega\right\} .
\end{gathered}
$$

Moreover, let $\chi_{\varepsilon}$ be the characteristic function of the set $\mathcal{D}_{\varepsilon}\left(x^{0}\right)$, i.e. $\chi_{\varepsilon}\left(x^{0}, \cdot\right): \partial \Omega_{\varepsilon}\left(x^{0}\right) \rightarrow\{0,1\}$ such that

$$
\chi_{\varepsilon}\left(x^{0}, y\right)=1 \text { iff } y \in \mathcal{D}_{\varepsilon}\left(x^{0}\right) .
$$

Note that whatever a positive constant $L$ is, $\Omega_{\varepsilon}\left(x^{0}\right) \cap B(L)$ approximately equals the half ball $B_{+}(L)$ for small enough $\varepsilon>0$. Recall that the small parameter $\varepsilon$ shall finally tend to zero and therefore $L \in \mathbb{R}_{+}$can be taken arbitrarily large.

We henceforth assume the following structure assumptions concerning the set $\mathcal{D}_{\varepsilon}\left(x^{0}\right)$, or equivalently, its characteristic function $\chi_{\varepsilon}$. 
Hypothesis (H). There exists a relatively closed set $\Gamma \subset \partial \Omega$ such that $\mathcal{H}^{n-1}(\Gamma)=0$ and for any $x^{0} \in \mathcal{G} \equiv \partial \Omega \backslash \Gamma$ there exist a function

$$
\chi: \mathbb{R}^{n-1} \rightarrow\{0,1\},
$$

a vector $\tau_{\varepsilon} \equiv\left(\tau_{\varepsilon}^{\prime}, 0\right) \in \mathbb{R}^{n}$ and a Lipschitz one-to-one map

$$
\Phi_{\varepsilon}: \bar{\Omega}_{\varepsilon}\left(x^{0}\right) \cap B(L) \longrightarrow \overline{\mathbb{R}}_{+}^{n}, \quad \Phi_{\varepsilon}(0)=0
$$

with the following five properties:

(i) The set $\mathbb{D} \equiv\left\{y^{\prime} \in \mathbb{R}^{n-1} \mid \chi\left(y^{\prime}\right)=1\right\}$ is asymptotically dense in $\mathbb{R}^{n-1}$.

(ii) Vectors $\tau_{\varepsilon}^{\prime}$ are uniformly bounded with respect to $\varepsilon$, i.e. $\left|\tau_{\varepsilon}^{\prime}\right| \leq m$ for some given positive constant $m$ independent of $\varepsilon$.

(iii)

$$
\Phi_{\varepsilon}: \partial \Omega_{\varepsilon}\left(x^{0}\right) \cap B(L) \longrightarrow \mathbb{R}^{n-1} \times\left\{z_{n}=0\right\} .
$$

(iv)

$$
\text { ess } \sup _{z \in \mathcal{R}_{\varepsilon}(L)}\left\|D \Phi_{\varepsilon}\left(\Psi_{\varepsilon}(z)\right)-\boldsymbol{I}\right\| \longrightarrow 0 \quad \text { as } \quad \varepsilon \rightarrow 0
$$

uniformly in $\mathcal{G}$, where

$$
\Psi_{\varepsilon} \equiv \Phi_{\varepsilon}^{-1}, \quad \mathcal{R}_{\varepsilon}(L) \equiv\left\{z \in \overline{\mathbb{R}}_{+}^{n} \mid z=\Phi_{\varepsilon}(y), y \in \bar{\Omega}_{\varepsilon}\left(x^{0}\right) \cap \bar{B}(L)\right\}
$$

and $\|\cdot\|$ denotes the matrix norm.

As the quantities $\chi, \tau_{\varepsilon}, \Phi_{\varepsilon}, \Psi_{\varepsilon}, \mathcal{R}_{\varepsilon}$ depend on $x^{0}$, we shall henceforth indicate this dependence writing $\chi\left(x^{0}, \cdot\right), \tau_{\varepsilon}\left(x^{0}\right), \Phi_{\varepsilon}\left(x^{0}, \cdot\right), \Psi_{\varepsilon}\left(x^{0}, \cdot\right)$ and $\mathcal{R}_{\varepsilon}\left(x^{0}, \cdot\right)$.

(v) For each $\kappa \in \mathbb{Z}_{+}$there exists a relatively closed set $\Gamma_{\kappa} \subset \partial \Omega$ such that

$$
\mathcal{H}^{n-1}\left(\Gamma_{\kappa}\right) \leq 1 / \kappa, \quad \Gamma_{\kappa+1} \subset \Gamma_{\kappa}, \quad \Gamma=\cap_{\kappa=1}^{\infty} \Gamma_{\kappa}
$$

and for fixed

$$
x^{0} \in \mathcal{G}^{\kappa} \equiv \partial \Omega \backslash \Gamma_{\kappa}
$$

define $H_{\varepsilon} \in W^{1,2}\left(B_{+}(R)\right), R<L$ to be a weak solution of the problem

$$
\begin{aligned}
\Delta H_{\varepsilon}=0 & \text { in } U_{R} \equiv B_{+}(R) \cap\left\{z \mid 0<z_{n}<1\right\}, \\
H_{\varepsilon}=0 & \text { on } B_{+}(R) \backslash U_{R}, \\
H_{\varepsilon}=1 & \text { on } \mathcal{P}_{\varepsilon}\left(x^{0}, R\right), \\
\partial_{\nu} H_{\varepsilon}=0 & \text { on } S_{R}\left(x^{0}\right),
\end{aligned}
$$


where

$$
\begin{aligned}
\mathcal{P}_{\varepsilon}\left(x^{0}, R\right) \equiv\left\{z \in B^{\prime}(R) \times\left\{z_{n}=0\right\} \mid\right. \\
\left.\left|\chi_{\varepsilon}\left(x^{0}, \Psi_{\varepsilon}\left(x^{0}, z\right)\right)-\chi\left(x^{0}, z^{\prime}+\tau_{\varepsilon}^{\prime}\left(x^{0}\right)\right)\right|=1\right\}
\end{aligned}
$$

and

$$
S_{R}\left(x^{0}\right) \equiv \partial U_{R} \backslash\left\{z \in \bar{B}_{+}(R) \mid z_{n}=1\right\} \cup \mathcal{P}_{\varepsilon}\left(x^{0}, R\right) .
$$

Then

$$
\text { ess } \sup _{x^{0} \in \mathcal{G}^{\kappa}} \int_{B_{+}(R)}\left|\nabla H_{\varepsilon}\right|^{2} d z \longrightarrow 0 \quad \text { as } \quad \varepsilon \rightarrow 0
$$

for any fixed $\kappa$.

Notation. For fixed $L \in \mathbb{R}_{+}$and $0<\varepsilon<1$ we define

$$
\ell_{\varepsilon}(L) \equiv \operatorname{ess} \sup _{x^{0} \in \partial \Omega}\left(\operatorname{ess} \sup _{z \in \mathcal{R}_{\varepsilon}\left(x^{0}, L\right)}\left\|D \Phi_{\varepsilon}\left(x^{0}, \Psi_{\varepsilon}\left(x^{0}, z\right)\right)-\boldsymbol{I}\right\|\right) .
$$

Remarks. (1) Note that for each $L \in \mathbb{R}_{+}$, (H) (iv) yields

$$
\lim _{\varepsilon \rightarrow 0} \ell_{\varepsilon}(L)=0
$$

and

$$
\bar{B}_{+}\left(L\left(1-\ell_{\varepsilon}\right)\right) \subset \mathcal{R}_{\varepsilon}\left(x^{0}, L\right) \subset \bar{B}_{+}\left(L\left(1+\ell_{\varepsilon}\right)\right)
$$

for any $x^{0} \in \partial \Omega$. Indeed, according to (iv)

$$
\Phi_{\varepsilon}(y)=y+\int_{0}^{1}\left(D \Phi_{\varepsilon}(s y)-\boldsymbol{I}\right) d s y
$$

for any $y \in \bar{\Omega}_{\varepsilon}\left(x^{0}\right) \cap B(L)$ such that $s y \in \bar{\Omega}_{\varepsilon}\left(x^{0}\right)$ for $s \in[0,1]$, that together with the Inverse Function Theorem give (1).

(2) The hypothesis $(v)$ is satisfied trivially if

$$
\chi_{\varepsilon}\left(x^{0}, \Psi_{\varepsilon}\left(x^{0}, z\right)\right)=\chi\left(x^{0}, z^{\prime}+\tau_{\varepsilon}^{\prime}\left(x^{0}\right)\right)
$$

for any $z \in \mathcal{R}_{\varepsilon}\left(x^{0}, L\right) \cap\left\{z \mid z_{n}=0\right\}$. 
Now we are ready to read off more detailed information concerning the weak limit of

$$
W_{\varepsilon} \equiv \frac{u_{\varepsilon}-u}{\varepsilon}
$$

on $\partial \Omega_{T}$ making use the blow-up of $\Omega$ around a point $x^{0} \in \partial \Omega$ (see [5, p. 198]). Recall first that $W_{\varepsilon}$ is the solution of

$$
\begin{aligned}
\partial_{t} W_{\varepsilon} & =\Delta W_{\varepsilon}+F_{\varepsilon}(x, t) & & \text { in } \Omega_{T}, \\
\partial_{\nu} W_{\varepsilon} & =\varepsilon^{-1} g_{\varepsilon}(x, t) & & \text { on } \mathcal{N}_{T}^{\varepsilon}, \\
W_{\varepsilon} & =0 & & \text { on } \mathcal{D}_{T}^{\varepsilon}, \\
W_{\varepsilon} & =\mathcal{P}_{\varepsilon} & & \text { on } \Omega \times\{t=0\},
\end{aligned}
$$

with

$$
g_{\varepsilon}=\vartheta_{\varepsilon}-\sigma_{\varepsilon} u_{\varepsilon}-\partial_{\nu} u
$$

in the following sense:

$$
\begin{array}{r}
\int_{\Omega} W_{\varepsilon}(\tau) \varphi_{\varepsilon}(\tau) d x-\int_{0}^{\tau} \int_{\Omega}\left(W_{\varepsilon} \partial_{t} \varphi_{\varepsilon}-\nabla W_{\varepsilon} \nabla \varphi_{\varepsilon}+F_{\varepsilon} \varphi_{\varepsilon}\right) d x d t \\
=\int_{\Omega} \mathcal{P}_{\varepsilon} \varphi_{\varepsilon}(0) d x+\varepsilon^{-1} \int_{0}^{\tau} \int_{\partial \Omega} g_{\varepsilon} \varphi_{\varepsilon} d H^{n-1}(x) d t
\end{array}
$$

holds for any $\varphi_{\varepsilon} \in W^{1,2}\left(\Omega_{T}\right), \varphi_{\varepsilon}=0$ on $\mathcal{D}_{T}^{\varepsilon}$.

Next fix $x^{0} \in \mathcal{G}$ and set

$$
w_{\varepsilon}\left(x^{0}, y, t\right) \equiv W_{\varepsilon}\left(x^{0}+\varepsilon \boldsymbol{O}\left(x^{0}\right) y, t\right)
$$

for $y \in \Omega_{\varepsilon}\left(x^{0}\right), t \in(0, T)$, and similarly $\eta_{\varepsilon}\left(x^{0}, y, t\right) \equiv \varphi_{\varepsilon}\left(x^{0}+\varepsilon \boldsymbol{O}\left(x^{0}\right) y, t\right)$. Moreover, take a test function $\varphi_{\varepsilon}$ in (4.7) such that

$$
\text { spt } \varphi_{\varepsilon} \subset B\left(x^{0}, \varepsilon L\right) \text {. }
$$

(4.7) then for $x=x^{0}+\varepsilon \boldsymbol{O}\left(x^{0}\right) y$ yields

$$
\begin{gathered}
\mathcal{O}_{\varepsilon}\left(x^{0}, s\right)+\int_{0}^{s} \int_{\Omega_{\varepsilon}\left(x^{0}\right) \cap B(L)} \nabla w_{\varepsilon} \nabla \eta_{\varepsilon} d y d t \\
=\int_{0}^{s} \int_{\partial \Omega_{\varepsilon}\left(x^{0}\right) \cap B(L)} g_{\varepsilon}\left(x^{0}+\varepsilon \boldsymbol{O} y, t\right) \eta_{\varepsilon}\left(x^{0}, y, t\right) d H^{n-1}(y) d t,
\end{gathered}
$$


where $\nabla$ means here the gradient in the $y$ variable and

$$
\begin{array}{r}
\mathcal{O}_{\varepsilon}\left(x^{0}, s\right) \equiv \varepsilon^{2} \int_{\Omega_{\varepsilon}\left(x^{0}\right) \cap B(L)}\left(w_{\varepsilon} \eta_{\varepsilon}(s)-\mathcal{P}_{\varepsilon}\left(x^{0}+\varepsilon \boldsymbol{O} y\right) \eta_{\varepsilon}(0)\right) d y \\
-\varepsilon^{2} \int_{0}^{s} \int_{\Omega_{\varepsilon}\left(x^{0}\right) \cap B(L)}\left(w_{\varepsilon} \partial_{t} \eta_{\varepsilon}+F_{\varepsilon}\left(x^{0}+\varepsilon \boldsymbol{O} y, t\right) \eta_{\varepsilon}\right) d y d t .
\end{array}
$$

Later on it will be not difficult to show that

$$
\int_{\partial \Omega} \mathcal{O}_{\varepsilon}\left(x^{0}, s\right) d H^{n-1}\left(x^{0}\right) \longrightarrow 0 \quad \text { as } \quad \varepsilon \downarrow 0
$$

for any $s \in(0, T)$ and therefore let us omit $\mathcal{O}_{\varepsilon}$ in further considerations.

Next we change variables in (4.9) utilizing Hypothesis (H) above and write

$$
z=\Phi_{\varepsilon}\left(x^{0}, y\right), \text { i.e. } y=\Psi_{\varepsilon}\left(x^{0}, z\right) .
$$

Then, after a little tedious, but not difficult manipulations we arrive at

$$
\begin{aligned}
\int_{0}^{s} \int_{\mathcal{G}^{\kappa}} \int_{\mathcal{R}_{\varepsilon}(L)} & \sum_{l, k=1}^{n} a_{\varepsilon}^{k l} \frac{\partial V_{\varepsilon}}{\partial z_{k}} \frac{\partial \xi_{\varepsilon}}{\partial z_{l}} d z d H^{n-1}\left(x^{0}\right) d t \\
= & \int_{0}^{s} \int_{\mathcal{G}^{\kappa}} \int_{\partial \mathcal{R}_{\varepsilon}(L)} G_{\varepsilon}\left(z^{\prime}\right) \eta\left(\hat{\Psi}_{\varepsilon}^{\prime}\left(z^{\prime}\right), t\right) \\
& \sqrt{1+\left|D \zeta_{\varepsilon}\left(\Psi_{\varepsilon}^{\prime}\left(z^{\prime}\right)\right)\right|^{2}} J \Psi_{\varepsilon}^{\prime}\left(z^{\prime}\right) d z^{\prime} d H^{n-1}\left(x^{0}\right) d t
\end{aligned}
$$

where

$$
\begin{gathered}
a_{\varepsilon}^{k l} \equiv J \Psi_{\varepsilon}(z) \sum_{i=1}^{n} \frac{\partial \Phi_{\varepsilon}^{k}}{\partial y_{i}}\left(\Psi_{\varepsilon}(z)\right) \frac{\partial \Phi_{\varepsilon}^{l}}{\partial y_{i}}\left(\Psi_{\varepsilon}(z)\right), \\
V_{\varepsilon}\left(x^{0}, z, t\right) \equiv w_{\varepsilon}\left(x^{0}, \Psi_{\varepsilon}\left(x^{0}, z\right), t\right), \quad \xi_{\varepsilon}\left(x^{0}, z, t\right) \equiv \eta_{\varepsilon}\left(x^{0}, \Psi_{\varepsilon}\left(x^{0}, z\right), t\right), \\
\hat{\Psi}_{\varepsilon}^{\prime} \equiv\left(\Psi_{\varepsilon}^{\prime}, \zeta_{\varepsilon}\left(\Psi_{\varepsilon}^{\prime}\right)\right), \quad \Psi_{\varepsilon}^{\prime}\left(z^{\prime}\right) \equiv\left(\Psi_{\varepsilon}^{1}\left(z^{\prime}, 0\right), \ldots, \Psi_{\varepsilon}^{n-1}\left(z^{\prime}, 0\right)\right), \\
\zeta_{\varepsilon}\left(y^{\prime}\right) \equiv \frac{1}{\varepsilon} \gamma\left(\varepsilon y^{\prime}\right), \quad G_{\varepsilon} \equiv g_{\varepsilon}\left(x^{0}+\varepsilon \boldsymbol{O}\left(x^{0}\right) \hat{\Psi}_{\varepsilon}^{\prime}\left(z^{\prime}\right), t\right),
\end{gathered}
$$

$\partial \mathcal{R}_{\varepsilon}(L) \equiv \Phi_{\varepsilon}\left(\partial \Omega_{\varepsilon}\left(x^{0}\right) \cap B(L)\right)$ and $\kappa$ being arbitrarily large.

Now let $\theta\left(x^{0}, z, t\right)$ be a sufficiently smooth function, say $\theta \in L^{2}\left(\partial \Omega_{T}\right.$; $\left.W_{\text {loc }}^{1,2}\left(\mathbb{R}_{+}^{n}\right)\right)$, such that

$$
\theta\left(x^{0}, z, t\right)=0 \quad \text { if } \quad z \in \mathbb{D}\left(x^{0}\right)
$$

and $\theta$ has a compact support lying in $\bar{B}_{+}(L-m)$, i.e.

$$
\operatorname{spt} \theta \subset \bar{B}_{+}(L-m) \text {. }
$$


Note that $\bar{B}_{+}(L-m) \subset \mathcal{R}_{\varepsilon}(L)$. Then

$$
\varphi_{\varepsilon}(x, t) \equiv \theta_{\varepsilon}\left(x^{0}, \Phi_{\varepsilon}\left(x^{0}, \boldsymbol{O}^{-1}\left(x^{0}\right) \frac{x-x^{0}}{\varepsilon}\right)+\tau_{\varepsilon}, t\right),
$$

where

$$
\theta_{\varepsilon}\left(x^{0}, z, t\right) \equiv \theta\left(x^{0}, z, t\right)\left(1-H_{\varepsilon}\left(x^{0}, z\right)\right)
$$

is admissible test function in (4.7), satisfying spt $\varphi_{\varepsilon} \subset \bar{B}\left(x^{0}, \varepsilon L\right)$, and corresponding test function in (4.12) has then the form

$$
\xi_{\varepsilon}\left(x^{0}, z, t\right)=\theta\left(x^{0}, z+\tau_{\varepsilon}, t\right)\left(1-H_{\varepsilon}\left(x^{0}, z+\tau_{\varepsilon}\right)\right)
$$

and one easily obtains

$$
\begin{gathered}
\int_{0}^{s} \int_{\mathcal{G}^{\kappa}} \int_{B_{+}(L-m)} \sum_{l, k=1}^{n} a_{\varepsilon}^{k l}\left(z-\tau_{\varepsilon}\right) \frac{\partial v_{\varepsilon}}{\partial z_{k}} \frac{\partial \theta_{\varepsilon}}{\partial z_{l}}\left(x^{0}, z, t\right) d z d H^{n-1}\left(x^{0}\right) d t \\
=\int_{0}^{s} \int_{\mathcal{G}^{\kappa}} \int_{B^{\prime}(L-m)} G_{\varepsilon}\left(z^{\prime}-\tau_{\varepsilon}^{\prime}\right) \theta_{\varepsilon}\left(x^{0}, z^{\prime}, 0, t\right) \\
\sqrt{1+\left|D \zeta_{\varepsilon}\left(\Psi_{\varepsilon}^{\prime}\left(z^{\prime}-\tau_{\varepsilon}^{\prime}\right)\right)\right|^{2}} J \Psi_{\varepsilon}^{\prime}\left(z^{\prime}-\tau_{\varepsilon}^{\prime}\right) d z^{\prime} d H^{n-1}\left(x^{0}\right) d t
\end{gathered}
$$

where

$$
v_{\varepsilon}\left(x^{0}, z, t\right) \equiv V_{\varepsilon}\left(x^{0}, z-\tau_{\varepsilon}, t\right) .
$$

If we let $\varepsilon \longrightarrow 0$, we arrive at

$$
\begin{aligned}
\int_{0}^{s} \int_{\mathcal{G}^{\kappa}} & \int_{B_{+}(L-m)} \nabla v \nabla \theta\left(x^{0}, z, t\right) d z d H^{n-1}\left(x^{0}\right) d t \\
& =\int_{0}^{s} \int_{\mathcal{G}^{\kappa}} \int_{B^{\prime}(L-m)} g\left(x^{0}, t\right) \theta\left(x^{0}, z^{\prime}, 0, t\right) d z^{\prime} d H^{n-1}\left(x^{0}\right) d t
\end{aligned}
$$

where

$$
g(x, t)=\vartheta(x, t)-\partial_{\nu} u(x, t)
$$

and where $v$ is a weak limit of $\left\{v_{\varepsilon}\right\}$. As $\kappa$ was choosen arbitrarily large, we can conclude that (4.14) holds also for $\mathcal{G}$ in place of $\mathcal{G}^{\kappa}$, i.e. for $\partial \Omega$.

This step will be verified in the next section. Now let us continue our consideration simply assuming that (4.14) holds for any sufficiently smooth 
test function $\theta$ with compact support (as the function of $z$ ) and such that $\theta=0$ on $\mathbb{D}$, and that the function $v$ satisfies the following a priori estimates:

$$
\begin{array}{r}
\int_{\partial \Omega_{T}} \int_{B^{\prime}(R) \times(0, R)}\left(1+z_{n}\right)|\nabla v|^{2}\left(x^{0}, z, t\right) d z d H^{n-1}\left(x^{0}\right) d t \leq C R^{n-1} \\
\int_{\partial \Omega_{T}} \int_{B^{\prime}(R)}|v|^{2}\left(x^{0}, z^{\prime}, 0, t\right) d z^{\prime} d H^{n-1}\left(x^{0}\right) d t \leq C R^{n-1}
\end{array}
$$

for any positive numbers $R$ greater than some given positive number $R_{0}$ and some positive constant $C$ independent of $R$.

We identify now the function $v$ determined by (4.14) and fulfilling (4.15).

Theorem 4.2. Assume $v \in L^{2}\left(\partial \Omega_{T} ; W_{\text {loc }}^{1,2}\left(\mathbb{R}_{+}^{n}\right)\right), v\left(x^{0}, z, t\right)=0$ if

$$
z \in \mathbb{D}_{0}\left(x^{0}\right) \equiv\left\{z=\left(z^{\prime}, 0\right) \in \mathbb{R}^{n} \mid \chi\left(x^{0}, z^{\prime}\right)=1\right\}
$$

for a.e. $\left(x^{0}, t\right) \in \partial \Omega_{T}$, and such that (4.14), (4.15) hold. Then

$$
v\left(x^{0}, z, t\right) \equiv g\left(x^{0}, t\right) \omega\left(x^{0}, z\right),
$$

where $\omega$ is the only bounded weak solution of the problem

$$
\begin{aligned}
\Delta_{z} \omega & =0 & & \text { in } \mathbb{R}_{+}^{n}, \\
\omega & =0 & & \text { on } \mathbb{D}_{0}, \\
\partial_{\nu} \omega & =1 & & \text { on } \mathbb{N}_{0},
\end{aligned}
$$

where $\mathbb{N} \equiv \mathbb{R}^{n-1} \backslash \mathbb{D}$.

Proof. 1. Using Fatou's Lemma and (4.15) we obtain

$$
\begin{aligned}
& \int_{\partial \Omega_{T}} \liminf _{R \rightarrow \infty} R^{1-n}\left\{\int_{B^{\prime}(R) \times(0, R)}|\nabla v|^{2}\left(1+z_{n}\right) d z\right. \\
&\left.+\int_{B^{\prime}(R)}|v|^{2}\left(z^{\prime}, 0\right) d z^{\prime}\right\} d H^{n-1}\left(x^{0}\right) d t \leq C,
\end{aligned}
$$

i.e. there exists a sequence $\left\{R_{i}\right\}_{i=1}^{\infty}, R_{i} \rightarrow \infty$ such that

$$
\begin{array}{r}
R_{i}^{1-n}\left\{\int_{B^{\prime}\left(R_{i}\right) \times\left(0, R_{i}\right)}|\nabla v|^{2}\left(1+z_{n}\right) d z+\int_{B^{\prime}\left(R_{i}\right)}|v|^{2}\left(z^{\prime}, 0\right) d z^{\prime}\right\} \\
\leq F\left(x^{0}, t\right)<\infty
\end{array}
$$

for a.e. $\left(x^{0}, t\right) \in \partial \Omega_{T}$, where $F \in L^{1}\left(\partial \Omega_{T}\right)$. Moreover, for a.e. $\left(x^{0}, t\right) \in$ $\partial \Omega_{T},(4.14)$ yields

$$
\int_{\mathbb{R}_{+}^{n}} \nabla v \nabla \psi d z=\int_{\mathbb{R}^{n-1}} g\left(x^{0}, t\right) \psi\left(z^{\prime}, 0\right) d z^{\prime}
$$


for any test function $\psi \in W_{l o c}^{1,2}\left(\mathbb{R}_{+}^{n}\right)$ with compact support and such that $\psi=0$ on $\mathbb{D}_{0}\left(x^{0}\right)$, i.e. $v$ is a weak solution of

$$
\begin{aligned}
\Delta_{z} v & =0 & & \text { in } \mathbb{R}_{+}^{n}, \\
v & =0 & & \text { on } \mathbb{D}_{0}, \\
\partial_{\nu} v & =g\left(x^{0}, t\right) & & \text { on } \mathbb{N}_{0} .
\end{aligned}
$$

2. Claim \#1. There exists positive constant $C=C\left(x^{0}, t\right)$, independent of $R \geq \max \{1, m\}$, such that

$$
f_{B^{\prime}(R) \times(0, R)}|v(z)| d z \leq C\left(1+(\ln R)^{1 / 2}\right) .
$$

Proof of Claim \#1. Note that for $1 \leq z_{n} \leq R$ we have

$$
\left|v\left(z^{\prime}, z_{n}\right)\right|-\left|v\left(z^{\prime}, 1\right)\right| \leq\left(\int_{1}^{R} \sigma\left|\frac{\partial v}{\partial z_{n}}\left(z^{\prime}, \sigma\right)\right|^{2} d \sigma\right)^{1 / 2}(\ln R)^{1 / 2}
$$

by Hölder inequality. Next integrate with respect to $z^{\prime}$ over $B^{\prime}(R)$ and with respect to $z_{n}$ over $(1, R)$ to find

$$
\begin{aligned}
& f_{B^{\prime}(R) \times(1, R)}|v(z)| d z \leq f_{B^{\prime}(R)}\left|v\left(z^{\prime}, 1\right)\right| d z^{\prime} \\
& \quad+\frac{1}{\alpha(n-1)}\left(\frac{1}{R^{n-1}} \int_{B^{\prime}(R) \times(1, R)} z_{n}\left|\frac{\partial v}{\partial z_{n}}\left(z^{\prime}, z_{n}\right)\right|^{2} d z\right)^{1 / 2}(\ln R)^{1 / 2} .
\end{aligned}
$$

To finish the proof we need only observe

$$
\begin{aligned}
f_{B^{\prime}(R)}\left|v\left(z^{\prime}, z_{n}\right)\right| d z^{\prime} \leq & f_{B^{\prime}(R)}\left|v\left(z^{\prime}, 0\right)\right| d z^{\prime} \\
& +\frac{1}{\alpha(n-1) R^{n-1}} \int_{B^{\prime}(R) \times(0,1)}\left|\frac{\partial v}{\partial z_{n}}(z)\right| d z
\end{aligned}
$$

for any $0 \leq z_{n} \leq 1$. Indeed, this and (4.18) yield

$$
f_{B^{\prime}\left(R_{i}\right) \times\left(0, R_{i}\right)}|v(z)| d z \leq 4 \sqrt{\frac{F\left(x^{0}, t\right)}{\alpha(n-1)}}\left(1+\sqrt{\ln R_{i}}\right) .
$$

This proves Claim \# 1 .

3. We know already that there is a bounded weak solution $v_{b} \equiv g\left(x^{0}, t\right) \omega$ of Problem (4.19) (see Theorem 3.4), i.e. $V \equiv v-v_{b}$ satisfies

$$
\begin{aligned}
\Delta V & =0 & & \text { in } \mathbb{R}_{+}^{n}, \\
V & =0 & & \text { on } \mathbb{D}_{0}, \\
\partial_{\nu} V & =0 & & \text { on } \mathbb{N}_{0},
\end{aligned}
$$


and

$$
f_{B_{+}\left(R_{i}\right)}|V(z)| d z \leq M\left(1+\sqrt{\ln R_{i}}\right)
$$

for any $i$. Hence, Theorem 3.3 in Sect. 3 implies $V \equiv 0$. This proves Theorem 4.2.

\section{A priori estimates}

We now ascertain the a priori estimates for $v_{\varepsilon}$,

$$
v_{\varepsilon}\left(x^{0}, z, t\right)=\frac{u_{\varepsilon}-u}{\varepsilon}\left(\Lambda_{\varepsilon}\left(x^{0}, z\right), t\right),
$$

where

$$
\Lambda_{\varepsilon}\left(x^{0}, z\right) \equiv x^{0}+\varepsilon \boldsymbol{O}\left(x^{0}\right) \Psi_{\varepsilon}\left(x^{0}, z-\tau_{\varepsilon}\left(x^{0}\right)\right),
$$

announced in the previous section. Note that for simplicity of notation we shall not always indicate the dependence of $\Lambda_{\varepsilon}, \boldsymbol{O}, \Psi_{\varepsilon}, v_{\varepsilon}$ on $x^{0}$.

Theorem 5.1. Let $(A)-(H)$ be satisfied. Then for any $R \in(m, \infty)$ there exists a positive $\varepsilon_{0}=\varepsilon_{0}(R)$ such that for any $\varepsilon \in\left(0, \varepsilon_{0}\right)$ the following estimates hold:

$$
I_{\varepsilon} \equiv \int_{\partial \Omega_{T}} \int_{\mathcal{B}(R)}\left|\nabla v_{\varepsilon}\right|^{2}\left(1+z_{n}\right) d z d H^{n-1}\left(x^{0}\right) d t \leq C R^{n-1},
$$

where $\nabla$ is the gradient in the $z$ variable,

$$
\mathcal{B}(R) \equiv \bar{B}^{\prime}(R) \times[0, R],
$$

and

$$
J_{\varepsilon} \equiv \int_{\partial \Omega_{T}} \int_{B^{\prime}(R)}\left|v_{\varepsilon}\left(z^{\prime}, 0\right)\right|^{2} d z^{\prime} d H^{n-1}\left(x^{0}\right) d t \leq C R^{n-1},
$$

wherein the positive constant $C$ does not depend on $\varepsilon, R$.

As a consequence of Theorem 5.1 we have

Corollary 5.2. There exists a function $v \in L^{2}\left(\partial \Omega_{T} ; W_{l o c}^{1,2}\left(\mathbb{R}_{+}^{n}\right)\right)$ and a subsequence of $\{\varepsilon\}$, denoting for simplicity again by $\varepsilon$, such that

$$
v_{\varepsilon} \rightarrow v \quad \text { in } \quad L^{2}\left(\partial \Omega_{T} ; W^{1,2}(Q)\right) \quad \text { as } \quad \varepsilon \downarrow 0
$$

for any relatively compact set $Q \subset \mathbb{R}_{+}^{n}$. Moreover, the estimates (4.15) and the integral identity (4.14) hold. 
Proof of Theorem 5.1. 1. Note first that

$$
\frac{\partial v_{\varepsilon}}{\partial z_{i}}=\sum_{j=1}^{n} a_{\varepsilon}^{i j}\left(\frac{\partial u_{\varepsilon}}{\partial x_{j}}-\frac{\partial u}{\partial x_{j}}\right)
$$

for $i=1, \ldots, n$, where

$$
a_{\varepsilon}^{i j} \equiv \sum_{k=1}^{n} o_{j k}\left(x^{0}\right) \frac{\partial \Psi_{\varepsilon}^{k}}{\partial z_{i}}\left(x^{0}, z-\tau_{\varepsilon}\right) .
$$

Then

$$
I_{\varepsilon} \leq c \int_{\partial \Omega_{T}} \int_{\mathcal{B}(R)}\left(1+z_{n}\right)\left|\nabla\left(u_{\varepsilon}-u\right)\right|^{2}\left(\Lambda_{\varepsilon}\left(x^{0}, z\right), t\right) d z d H^{n-1}\left(x^{0}\right) d t .
$$

Put now

$$
x=\Lambda_{\varepsilon}\left(x^{0}, z\right), \text { i.e. } z=\Lambda_{\varepsilon}^{-1}\left(x^{0}, x\right)
$$

and we get

$$
\begin{aligned}
I_{\varepsilon} & \leq C_{1} \int_{\partial \Omega_{T}} \int_{\Lambda_{\varepsilon}\left(x^{0}, \mathcal{B}\right)}\left|\nabla\left(u_{\varepsilon}-u\right)\right|^{2} \\
& \leq C_{2} \varepsilon^{-n} \int_{\partial \Omega_{T}} \int_{\Lambda_{\varepsilon}\left(x^{0}, \mathcal{B}\right)}\left|\nabla\left(u_{\varepsilon}-u\right)\right|^{2}\left(1+\varepsilon^{-1} \phi\right) d x d H^{n-1}\left(x^{0}\right) d t,
\end{aligned}
$$

where

$$
\Lambda_{\varepsilon}\left(x^{0}, \mathcal{B}(R)\right) \equiv\left\{x \in \bar{\Omega} \mid x=\Lambda_{\varepsilon}\left(x^{0}, z\right), z \in \mathcal{B}(R)\right\} .
$$

Let $\chi_{\mathcal{B}}^{\varepsilon}\left(x^{0}, x\right)$ be the indicator function of the set $\Lambda_{\varepsilon}\left(x^{0}, \mathcal{B}(R)\right)$, i.e.

$$
\chi_{\mathcal{B}}^{\varepsilon}\left(x^{0}, x\right)=\left\{\begin{array}{lll}
1 & : & x \in \Lambda_{\varepsilon}\left(x^{0}, \mathcal{B}(R)\right) \\
0 & : & x \in \bar{\Omega} \backslash \Lambda_{\varepsilon}\left(x^{0}, \mathcal{B}(R)\right)
\end{array} .\right.
$$

Then the above inequality can be rewritten as follows:

$$
\begin{aligned}
I_{\varepsilon} & \leq C_{3} \varepsilon^{-n} \int_{\partial \Omega_{T}} \int_{\Omega}\left|\nabla\left(u_{\varepsilon}-u\right)\right|^{2}\left(1+\varepsilon^{-1} \phi\right) \chi_{\mathcal{B}}^{\varepsilon}\left(x^{0}, x\right) d x d H^{n-1}\left(x^{0}\right) d t \\
& =C_{3} \varepsilon^{-n} \int_{\Omega_{T}}\left|\nabla\left(u_{\varepsilon}-u\right)\right|^{2}\left(1+\varepsilon^{-1} \phi\right) \int_{\partial \Omega} \chi_{\mathcal{B}}^{\varepsilon}\left(x^{0}, x\right) d H^{n-1}\left(x^{0}\right) d x d t
\end{aligned}
$$

by Fubini's theorem. It remains to estimate the integral

$$
\int_{\partial \Omega} \chi_{\mathcal{B}}^{\varepsilon}\left(x^{0}, x\right) d H^{n-1}\left(x^{0}\right) \quad \text { for fixed } x \in \bar{\Omega} .
$$


2. Claim \#1: There exists a positive constant $M$, independent of $x, \varepsilon, R$ and a positive constant $\varepsilon_{0}=\varepsilon_{0}(R)$ such that

$$
\int_{\partial \Omega} \chi_{\mathcal{B}}^{\varepsilon}\left(x^{0}, x\right) d H^{n-1}\left(x^{0}\right) \leq M(R+m)^{n-1} \varepsilon^{n-1}
$$

for any $\varepsilon \in\left(0, \varepsilon_{0}\right]$.

Proof of Claim \#1: Note first that $\Phi_{\varepsilon}\left(\Psi_{\varepsilon}(z)\right)=z$ and that $D \Phi_{\varepsilon}\left(\Psi_{\varepsilon}(z)\right)$ $D \Psi_{\varepsilon}(z)=\boldsymbol{I}$ for $\mathcal{L}^{n}$ a.e. $z \in \mathcal{R}_{\varepsilon}(L)$, where we take $L \gg R+m$ and for a moment we do not indicate the dependence of the quantities on $x^{0}$. Next, $D \Phi_{\varepsilon}\left(\Psi_{\varepsilon}(z)\right)=\boldsymbol{I}+\boldsymbol{E}_{\varepsilon}(z)$ for "small" $\boldsymbol{E}_{\varepsilon}$ (see Remark (1) above) and

$$
D \Psi_{\varepsilon}(z)=\boldsymbol{I}+\boldsymbol{F}_{\varepsilon}(z), \boldsymbol{F}_{\varepsilon}(z) \equiv \boldsymbol{E}_{\varepsilon}(z)\left(D \Phi_{\varepsilon}\right)^{-1}\left(\Psi_{\varepsilon}(z)\right) .
$$

Denoting

$$
\begin{gathered}
a_{\varepsilon}(L) \equiv \operatorname{ess} \sup _{x^{0} \in \partial \Omega}\left(\operatorname{ess} \sup _{z \in \mathcal{R}_{\varepsilon}\left(x^{0}, L\right)}\left\|\boldsymbol{F}_{\varepsilon}\left(x^{0}, z\right)\right\|\right), \\
\lim _{\varepsilon \rightarrow 0} a_{\varepsilon}(L)=0 .
\end{gathered}
$$

Hence,

$$
\Psi_{\varepsilon}\left(x^{0}, z\right)=\left(\boldsymbol{I}+\int_{0}^{1} \boldsymbol{F}_{\varepsilon}\left(x^{0}, s z\right) d s\right) z
$$

for any $z \in B_{+}\left(L\left(1-\ell_{\varepsilon}(L)\right)\right)$. Observe now that for fixed $x \in \bar{\Omega}, \chi_{\mathcal{B}}^{\varepsilon}\left(x^{0}, x\right)$ could equal 1 only for those $x^{0} \in \partial \Omega$ for which

$$
x=x^{0}+\varepsilon \boldsymbol{O}\left(x^{0}\right) \Psi_{\varepsilon}\left(x^{0}, z-\tau_{\varepsilon}\left(x^{0}\right)\right)
$$

for some $z \in \mathcal{B}(R)$. Hence, due to (5.4), $\chi_{\mathcal{B}}^{\varepsilon}\left(x^{0}, x\right)$ could be 1 only if

$$
\left|x-x^{0}\right| \leq \varepsilon(\sqrt{2} R+m)\left(1+a_{\varepsilon}\right) .
$$

Then, however,

$$
\begin{aligned}
& \int_{\partial \Omega} \chi_{\mathcal{B}}^{\varepsilon}\left(x^{0}, x\right) d H^{n-1}\left(x^{0}\right) \leq \mathcal{H}^{n-1}\left(\partial \Omega \cap B\left(x, \varepsilon L_{\varepsilon}\right)\right) \\
& \leq \int_{B^{\prime}\left(\varepsilon L_{\varepsilon}\right)} \sqrt{1+\left|D \gamma\left(x, y^{\prime}\right)\right|^{2}} d y^{\prime} \leq\left(1+c_{\varepsilon}(L)\right) \alpha(n-1) \varepsilon^{n-1} L_{\varepsilon}^{n-1},
\end{aligned}
$$

where $L_{\varepsilon} \equiv(\sqrt{2} R+m)\left(1+a_{\varepsilon}\right)$ and

$$
c_{\varepsilon}(L) \equiv \operatorname{ess} \sup _{x \in \partial \Omega}\left(\text { ess } \sup _{y^{\prime} \in B^{\prime}(\varepsilon L)}\left|D \gamma\left(x, y^{\prime}\right)\right|^{2}\right) .
$$


As $\gamma \in C^{2}$ and $D \gamma(x, 0)=0$,

$$
\lim _{\varepsilon \rightarrow 0} c_{\varepsilon}(L)=0
$$

and the required assertion follows.

3. To derive estimate (5.2) we shall proceed similarly as above. Note first

$$
J_{\varepsilon}=\int_{\partial \Omega_{T}} \int_{B^{\prime}(R)}\left|\frac{u_{\varepsilon}-u}{\varepsilon}\left(x^{0}+\varepsilon \boldsymbol{O}\left(x^{0}\right) \hat{\Psi}_{\varepsilon}^{\prime}\left(z^{\prime}-\tau_{\varepsilon}^{\prime}, t\right)\right)\right|^{2} d z^{\prime} d H^{n-1}\left(x^{0}\right) d t
$$

where

$$
\varepsilon \hat{\Psi}_{\varepsilon}^{\prime}\left(z^{\prime}-\tau_{\varepsilon}^{\prime}\right)=\left(\varepsilon \Psi_{\varepsilon}^{\prime}\left(x^{0}, z^{\prime}-\tau_{\varepsilon}^{\prime}, 0\right), \gamma\left(\varepsilon \Psi_{\varepsilon}^{\prime}\left(x^{0}, z^{\prime}-\tau_{\varepsilon}^{\prime}, 0\right)\right)\right) .
$$

Put now

$$
y^{\prime}=\varepsilon \Psi_{\varepsilon}^{\prime}\left(z^{\prime}-\tau_{\varepsilon}^{\prime}, 0\right), \text { i.e. } \quad z^{\prime}=\tau_{\varepsilon}^{\prime}+\Phi_{\varepsilon}^{\prime}\left(x^{0}, \varepsilon^{-1} y^{\prime}, \gamma\left(\varepsilon^{-1} y^{\prime}\right)\right)
$$

and we arrive, after some computations, at

$$
J_{\varepsilon} \leq C_{1} \varepsilon^{1-n} \int_{\partial \Omega_{T}} \int_{\partial \Omega}\left|\frac{u_{\varepsilon}-u}{\varepsilon}\right|^{2}(x, t) \chi_{U}^{\varepsilon}\left(x^{0}, x\right) d H^{n-1}(x) d H^{n-1}\left(x^{0}\right) d t,
$$

where

$$
\chi_{U}^{\varepsilon}\left(x^{0}, x\right)=\left\{\begin{array}{lll}
1 & : & x \in U\left(x^{0}, \varepsilon R\right) \\
0 & : & x \in \partial \Omega \backslash U\left(x^{0}, \varepsilon R\right)
\end{array}\right.
$$

and

$$
U\left(x^{0}, \varepsilon R\right) \equiv\left\{x \in \partial \Omega \mid x=\Lambda_{\varepsilon}\left(x^{0}, z\right) \quad \text { for } \quad z \in B^{\prime}(R) \times\left\{z_{n}=0\right\}\right\} .
$$

Thus, by Fubini's theorem,

$$
J_{\varepsilon} \leq C_{1} \varepsilon^{1-n} \int_{\partial \Omega_{T}}\left|\frac{u_{\varepsilon}-u}{\varepsilon}\right|^{2}(x, t) \int_{\partial \Omega} \chi_{U}^{\varepsilon}\left(x^{0}, x\right) d H^{n-1}\left(x^{0}\right) d H^{n-1}(x) d t .
$$

Now, let $x \in \partial \Omega$ be fixed. Then

$$
\chi_{U}^{\varepsilon}\left(x^{0}, x\right)=0 \quad \text { if } \quad x^{0} \in \partial \Omega \backslash B\left(x, \varepsilon(R+m)\left(1+a_{\varepsilon}\right)\right),
$$

i.e.

$$
\begin{aligned}
\int_{\partial \Omega} \chi_{U}^{\varepsilon}\left(x^{0}, x\right) d H^{n-1}\left(x^{0}\right) & \\
& \leq\left(1+c_{\varepsilon}(L)\right) \alpha(n-1)\left(\varepsilon(R+m)\left(1+a_{\varepsilon}(L)\right)\right)^{n-1}
\end{aligned}
$$


(see the proof of Claim \#1 above). Hence,

$$
J_{\varepsilon} \leq C_{3} R^{n-1} \int_{\partial \Omega_{T}}\left|\frac{u_{\varepsilon}-u}{\varepsilon}\right|^{2}(x, t) d H^{n-1}(x) d t,
$$

which together with the corresponding estimate of Theorem 2.1 gives the desired estimate.

Proof of Corollary 5.2. Observe first that for any $R \in \mathbb{R}_{+}$(5.1) and (5.2) yields

$$
\int_{\partial \Omega_{T}} \int_{B_{+}(R)}\left(\left|\nabla v_{\varepsilon}\right|^{2}+\left|v_{\varepsilon}\right|^{2}\right) d z d H^{n-1}\left(x^{0}\right) d t \leq C(R),
$$

provided $\varepsilon$ is sufficiently small. To be more precise, for any $R$ there exists $\varepsilon_{0}=\varepsilon_{0}(R)$ such that

$$
\left\|v_{\varepsilon}\right\|_{X(R)} \leq C(R), \quad X(R) \equiv L^{2}\left(\partial \Omega_{T} ; W^{1,2}\left(B_{+}(R)\right)\right)
$$

for any $0<\varepsilon \leq \varepsilon_{0}$. Now let $\left\{R_{n}\right\}_{n=1}^{\infty}$ be a sequence of positive numbers such that $R_{n} \rightarrow \infty$ as $n \rightarrow \infty$. By the relative weak compactness of the sequence $\left\{v_{\varepsilon}\right\}$ in $X\left(R_{1}\right)$ we can pick a subsequence $\left\{v_{1 \varepsilon_{n}}\right\}_{n=1}^{\infty}$ such that $v_{1 \varepsilon_{n}}$ weakly converges to a function $v \in X\left(R_{1}\right)$. We can now pick a subsequence $\left\{v_{2 \varepsilon_{n}}\right\}_{n=1}^{\infty}$ of $\left\{v_{1 \varepsilon_{n}}\right\}_{n=1}^{\infty}$ such that $v_{2 \varepsilon_{n}}$ weakly converges to $v^{\prime}$ in $X\left(R_{2}\right)$. Clearly,

$$
v^{\prime} \equiv v \quad \text { on } \quad B_{+}\left(R_{1}\right)
$$

and therefore, we shall write $v$ instead of $v^{\prime} \in B_{+}\left(R_{2}\right)$. Continuing in this fashion we obtain a subsequence $\left\{v_{j \varepsilon_{n}}\right\}_{n=1}^{\infty}$ weakly convergent to $v$ in $X\left(R_{j}\right)$.

Consider now the "diagonal" sequence $\left\{v_{n \varepsilon_{n}}\right\}_{n=1}^{\infty}$. We have $\left\{v_{n \varepsilon_{n}}\right\}_{n=j}^{\infty}$ a subsequence of $\left\{v_{j \varepsilon_{n}}\right\}_{n=1}^{\infty}$ and so $\left\{v_{n \varepsilon_{n}}\right\}$ weakly converges to $v$ in $X\left(R_{j}\right)$ for any fixed $j$. This establishes the first assertion of Corollary 5.2, and the second one follows easily.

\section{The second term in the $\varepsilon$-expansion}

As noted earlier, we are interested in specifying the second term in the $\varepsilon$-expansion of $u_{\varepsilon}$. Assume for a moment $\zeta \in C_{2}^{2,1}\left(\bar{\Omega}_{T}\right), \zeta=0$ on $\partial \Omega_{T}$, and $\zeta=0$ on $\Omega \times\{t=T\}$, and insert it as a test function into (4.7). Consequently, we have

$$
\begin{aligned}
\int_{\partial \Omega_{T}} W_{\varepsilon} \partial_{\nu} \zeta\left(x^{0}, t\right) d H^{n-1}\left(x^{0}\right) d t & \\
= & \int_{\Omega_{T}} W_{\varepsilon}\left(\partial_{t} \zeta+\Delta \zeta+F_{\varepsilon}\right) d x d t-\int_{\Omega} \mathcal{P}_{\varepsilon} \zeta(0) d x .
\end{aligned}
$$


Send $\varepsilon \rightarrow 0-$ through a subsequence if necessary - to find

$$
\begin{aligned}
& \int_{\partial \Omega_{T}} W_{0} \partial_{\nu} \zeta d H^{n-1}\left(x^{0}\right) d t \\
&=\int_{\Omega_{T}} u^{1}\left(\partial_{t} \zeta+\Delta \zeta+f^{1}\right) d x d t-\int_{\Omega} u_{0}^{1} \zeta(0) d x
\end{aligned}
$$

where our hypotheses $(\mathrm{C})-(\mathrm{F})$ have been applied and

$$
W_{0}, u^{1} \text { are weak limits of } W_{\varepsilon}
$$

in $L^{2}\left(\partial \Omega_{T}\right), L^{2}\left(\Omega_{T}\right)$, respectively. Note that $u^{1} \in L^{2}\left(\Omega_{T}\right) \cap W_{2}^{2,1}\left(\Omega_{T}^{\prime}\right)$ for any $\Omega^{\prime} \subset \Omega$ with a positive distance from $\partial \Omega$ and such that

$$
\begin{gathered}
\int_{\Omega_{T}}\left|u^{1}\right|^{2} d x d t+\max _{0 \leq t \leq T} \int_{\Omega}\left|u^{1}(t)\right|^{2} \phi d x+\int_{\Omega_{T}}\left|\nabla u^{1}\right|^{2} \phi d x d t \leq C, \\
\text { ess } \sup _{0 \leq t \leq T} \int_{\Omega}\left|\nabla u^{1}(t)\right|^{2} \phi^{3} d x+\int_{\Omega_{T}}\left|\partial_{t} u^{1}\right|^{2} \phi^{3} d x d t \leq C .
\end{gathered}
$$

Moreover, $u^{1}$ is the unique very weak solution of the problem

$$
\begin{aligned}
\partial_{t} u^{1} & =\Delta u^{1}+f^{1} & & \text { in } \Omega_{T}, \\
u^{1} & =W_{0} & & \text { on } \partial \Omega_{T}, \\
u^{1} & =u_{0}^{1} & & \text { on } \Omega \times\{t=0\}
\end{aligned}
$$

in the sense of the validity of the integral identity (6.2) for any test function $\zeta \in W_{2}^{2,1}\left(\Omega_{T}\right)$ such that $\zeta=0$ on $\partial \Omega_{T}$. It is not difficult to show that if $\hat{u}^{1}$ is another very weak solution of (6.3) with $\hat{W}_{0}$ and $\hat{u}_{0}^{1}$, then

$$
\int_{\Omega_{T}}\left|u^{1}-\hat{u}^{1}\right| d x d t \leq C\left(\left\|W_{0}-\hat{W}_{0}\right\|_{L^{2}\left(\partial \Omega_{T}\right)}+\left\|u_{0}^{1}-\hat{u}_{0}^{1}\right\|_{L^{2}(\Omega)}\right) .
$$

Since our goal is to determine the function $W_{0}$, we shall focus the first term of (6.1).

Theorem 6.1. Let Hypothesis $(H)$ be satisfied and assume that

$$
\lim _{R \rightarrow \infty} f_{B^{\prime}(R)} \omega\left(x^{0}, z^{\prime}, 0\right) d z^{\prime}
$$

exists for a.e. $x^{0} \in \partial \Omega$. We shall denote it by $M\left(x^{0}\right)$ and suppose that $M \in L^{\infty}(\partial \Omega)$. Then

$$
\lim _{\varepsilon \rightarrow 0} \int_{\partial \Omega_{T}} W_{\varepsilon} \partial_{\nu} \zeta=\int_{\partial \Omega_{T}} M\left(x^{0}\right) g\left(x^{0}, t\right) \partial_{\nu} \zeta\left(x^{0}, t\right) d H^{n-1}\left(x^{0}\right) d t
$$

(through a subsequence if necessary) for any $\zeta \in W_{2}^{2,1}\left(\Omega_{T}\right)$. 
Proof of Theorem 6.1. We prove that for $\mathcal{H}^{n-1}$ a.e. $x \in \partial \Omega$,

$$
W_{0}(x, t)=M(x) g(x, t), \quad g(x, t)=\vartheta(x, t)-\partial_{\nu} u(x, t) .
$$

To this goal, fix arbitrarily choosen $X \in \mathcal{G}$ and note that there exist $\iota>0$ and $\kappa \in \mathbb{Z}_{+}$such that

$$
B(X, 2 \iota) \cap \partial \Omega \subset \mathcal{G}^{\kappa} .
$$

Recall that $\mathcal{G}, \mathcal{G}^{\kappa}$ are introduced in Hypothesis (H) in Sect. 4. We take now $\varphi \in C\left(\bar{\Omega}_{T}\right)$ such that spt $\varphi \cap \partial \Omega \subset B(X, 2 \iota)$, but arbitrary otherwise, and show that for any $\eta>0$ there exists $\varepsilon_{0}(\eta)>0$ such that

$$
\int_{\partial \Omega_{T}}\left(W_{\varepsilon}\left(x^{0}, t\right)-M\left(x^{0}\right) g\left(x^{0}, t\right)\right) \varphi\left(x^{0}, t\right) d H^{n-1}\left(x^{0}\right) d t<\eta
$$

for any $0<\varepsilon \leq \varepsilon_{0}$.

Denote

$$
\begin{aligned}
I_{\varepsilon}+I I_{\varepsilon} \equiv & \int_{\partial \Omega_{T}}\left(W_{\varepsilon}\left(x^{0}, t\right)\right. \\
& \left.-\int_{U\left(x^{0}, \varepsilon R\right)} W_{\varepsilon}(x, t) d H^{n-1}(x)\right) \varphi\left(x^{0}, t\right) d H^{n-1}\left(x^{0}\right) d t \\
& +\int_{\partial \Omega_{T}} f_{U\left(x^{0}, \varepsilon R\right)} W_{\varepsilon}(x, t) d H^{n-1}(x) \varphi\left(x^{0}, t\right) d H^{n-1}\left(x^{0}\right) d t,
\end{aligned}
$$

where the set $U\left(x^{0}, \varepsilon R\right)$ is given by (5.6) above. Denoting the characteristic function of the set $U\left(x^{0}, \varepsilon R\right)$ by $\chi_{U}^{\varepsilon}\left(x^{0}, x\right)$ (see (5.6) above), using Fubini's theorem and relabeling the integration variable in the first term of $I_{\varepsilon}$ we arrive at

$$
I_{\varepsilon}=\int_{\partial \Omega_{T}} W_{\varepsilon}(x, t)\left(\varphi(x, t)-\varphi_{\varepsilon}(x, t)\right) d H^{n-1}(x) d t,
$$

where

$$
\varphi_{\varepsilon}(x, t) \equiv \int_{\partial \Omega} \frac{\chi_{U}^{\varepsilon}\left(x^{0}, x\right) \varphi\left(x^{0}, t\right)}{\mathcal{H}^{n-1}\left(U\left(x^{0}, \varepsilon R\right)\right)} d H^{n-1}\left(x^{0}\right) .
$$

Claim \#1: For any $R \in \mathbb{R}_{+}$and $\varepsilon \in(0,1)$ there exists a positive constant $\varpi_{\varepsilon}(R)$ such that

$$
\lim _{\varepsilon \rightarrow 0} \varpi_{\varepsilon}(R)=0
$$

and

$$
\left|\frac{\mathcal{H}^{n-1}\left(U\left(x^{0}, \varepsilon R\right)\right)}{\mathcal{L}^{n-1}\left(B^{\prime}(\varepsilon R)\right)}-1\right| \leq \frac{m}{R}+\varpi_{\varepsilon}(R)
$$

for any $x^{0} \in \mathcal{G}$. 
Proof of Claim \#1: As $\left|\tau_{\varepsilon}\right| \leq m$,

$$
U(R-m) \subset U\left(x^{0}, \varepsilon R\right) \subset U(R+m),
$$

where

$$
U(N) \equiv\left\{x \in \partial \Omega \mid x=x^{0}+\varepsilon \boldsymbol{O}\left(x^{0}\right) \hat{\Psi}_{\varepsilon}^{\prime}\left(x^{0}, z^{\prime}\right) \text { for } z^{\prime} \in B^{\prime}(N)\right\} .
$$

We claim now that

$$
\mathcal{H}^{n-1}(U(N))=\mathcal{L}^{n-1}\left(B^{\prime}(\varepsilon N)\right)\left(1+h_{\varepsilon}\left(x^{0}, N\right)\right), \quad \lim _{\varepsilon \rightarrow 0} h_{\varepsilon}\left(x^{0}, N\right)=0
$$

uniformly in $x^{0} \in \mathcal{G}$ for any fixed $N$. Indeed,

$$
\begin{aligned}
\mathcal{H}^{n-1}(U(N)) & =\int_{\partial \Omega \cap B\left(x^{0}, r\right)} \chi_{U(N)}(x) d H^{n-1}(x) \\
& =\varepsilon^{n-1} \int_{B^{\prime}(N)} \sqrt{1+\left|D \gamma\left(x^{0}, \varepsilon \Psi_{\varepsilon}^{\prime}\left(x^{0}, z^{\prime}\right)\right)\right|^{2}} J \Psi_{\varepsilon}^{\prime}\left(x^{0}, z^{\prime}\right) d z^{\prime}
\end{aligned}
$$

and the assertion follows easily due to (5.3) and the fact that $D \gamma\left(x^{0}, 0\right)=0$ for any $x^{0} \in \partial \Omega$. Thus,

$$
\begin{aligned}
\left(1-\frac{m}{R}\right)^{n-1}\left(1+h_{\varepsilon}\left(x^{0}, R-m\right)\right) & \leq \frac{\mathcal{H}^{n-1}\left(U\left(x^{0}, \varepsilon R\right)\right)}{\mathcal{L}^{n-1}\left(B^{\prime}(\varepsilon R)\right)} \\
& \leq\left(1+\frac{m}{R}\right)^{n-1}\left(1+h_{\varepsilon}\left(x^{0}, R+m\right)\right),
\end{aligned}
$$

which proves (6.7) after some manipulations. This proves Claim \# 1.

Hence,

$$
\begin{gathered}
\varphi_{\varepsilon}(x, t)=\frac{\varphi(x, t)}{\mathcal{L}^{n-1}\left(B^{\prime}(\varepsilon R)\right)} \int_{\partial \Omega} \chi_{U}^{\varepsilon}\left(x^{0}, x\right) d H^{n-1}\left(x^{0}\right)+ \\
\frac{1}{\mathcal{L}^{n-1}\left(B^{\prime}(\varepsilon R)\right)} \int_{\partial \Omega} \chi_{U}^{\varepsilon}\left(x^{0}, x\right) \frac{\varphi\left(x^{0}, t\right)-\varphi(x, t)-\theta_{\varepsilon}\left(x^{0}, R\right)}{1+\theta_{\varepsilon}\left(x^{0}, R\right)} d H^{n-1}\left(x^{0}\right),
\end{gathered}
$$

where

$$
\theta_{\varepsilon}\left(x^{0}, R\right) \equiv \frac{\mathcal{H}^{n-1}\left(U\left(x^{0}, \varepsilon R\right)\right)}{\mathcal{L}^{n-1}\left(B^{\prime}(\varepsilon R)\right)}-1
$$

and due to Claim \# 1,

$$
\left|\theta_{\varepsilon}\left(x^{0}, R\right)\right| \leq \frac{m}{R}+\varpi_{\varepsilon}(R) .
$$


Claim \#2: For any $R \in \mathbb{R}_{+}$and $\varepsilon \in(0,1)$ there exists a positive constant $\mu_{\varepsilon}(R)$ such that

$$
\lim _{\varepsilon \rightarrow 0} \mu_{\varepsilon}(R)=0
$$

and

$$
\left|\frac{\mathcal{H}^{n-1}(\mathcal{F}(x))}{\mathcal{L}^{n-1}\left(B^{\prime}(\varepsilon R)\right)}-1\right| \leq \frac{m}{R}+\mu_{\varepsilon}(R)
$$

for any $x \in \mathcal{G}$, where

$$
\mathcal{F}(x) \equiv\left\{x^{0} \in \partial \Omega \mid x \in U\left(x^{0}, \varepsilon R\right)\right\} .
$$

Proof of Claim \#2: Note first that

$$
\mathcal{H}^{n-1}(\mathcal{F}(x))=\int_{\partial \Omega \cap B\left(x^{0}, r\right)} \chi_{U}^{\varepsilon}\left(x^{0}, x\right) d H^{n-1}\left(x^{0}\right)
$$

for small enough $\varepsilon>0$ and denote for a moment

$$
\begin{array}{ll}
\mathcal{A}(x) \equiv\left\{x^{0} \in \partial \Omega|| x-x^{0} \mid \leq r_{\varepsilon}\right\}, & r_{\varepsilon} \equiv \varepsilon(R-m) /\left(1+\ell_{\varepsilon}(L)\right), \\
\mathcal{Z}(x) \equiv\left\{x^{0} \in \partial \Omega|| x-x^{0} \mid \leq R_{\varepsilon}\right\}, & R_{\varepsilon} \equiv \varepsilon(R+m)\left(1+a_{\varepsilon}(L)\right),
\end{array}
$$

where $\ell_{\varepsilon}$ is given in (4.5), $a_{\varepsilon}$ in (5.3) and $L>R+m$ being given.

Then $\mathcal{A}(x) \subset \mathcal{F}(x) \subset \mathcal{Z}(x)$ for any $x \in \mathcal{G}$ and thus,

$$
\mathcal{H}^{n-1}(\mathcal{A}(x)) \leq \mathcal{H}^{n-1}(\mathcal{F}(x)) \leq \mathcal{H}^{n-1}(\mathcal{Z}(x)) .
$$

Indeed, let $x^{0} \in \mathcal{A}(x)$. Setting $\left.z=\tau_{\varepsilon}\left(x^{0}\right)+\Phi_{\varepsilon}\left(x^{0}, \varepsilon^{-1} \boldsymbol{O}^{-1}\left(x^{0}\right)\left(x-x^{0}\right)\right)\right)$ one can see that

$$
|z| \leq m+\varepsilon^{-1}\left(1+\ell_{\varepsilon}(L)\right)\left|x-x^{0}\right| \leq R \text {, i.e. } \quad x^{0} \in \mathcal{F}(x) .
$$

Now, let $x^{0} \in \mathcal{F}(x)$, i.e. there exists $z \in B^{\prime}(R) \times\left\{z_{n}=0\right\}$ such that $x=\Lambda_{\varepsilon}\left(x^{0}, z\right)$. Then, however,

$$
\left|x-x^{0}\right| \leq R_{\varepsilon}, \quad \text { i.e. } \quad x^{0} \in \mathcal{Z}(x) .
$$

Next, it is not difficult to see that

$$
\mathcal{H}^{n-1}(\mathcal{Z}(x)) \leq\left(1+c_{\varepsilon}(L)\right) \mathcal{L}^{n-1}\left(B^{\prime}\left(R_{\varepsilon}\right)\right)
$$

and

$$
\mathcal{L}^{n-1}\left(B^{\prime}\left(r_{\varepsilon} / \sqrt{1+\operatorname{Lip}^{2}\left(\left.\gamma\right|_{B^{\prime}\left(r_{\varepsilon}\right)}\right)}\right)\right) \leq \mathcal{H}^{n-1}(\mathcal{A}(x)) .
$$

(6.8) follows again after some manipulations and Claim \# 2 is proved. 
Note that Claim \# 2 yields

$$
\int_{\partial \Omega} \chi_{U}^{\varepsilon}\left(x^{0}, x\right) d H^{n-1}\left(x^{0}\right)=\mathcal{L}^{n-1}\left(B^{\prime}(\varepsilon R)\right)\left(1+\xi_{\varepsilon}(x, R)\right),
$$

where

$$
\left|\xi_{\varepsilon}(x, R)\right| \leq \frac{m}{R}+\mu_{\varepsilon}(R)
$$

uniformly in $x \in \mathcal{G}$.

Now, with the assistance of the estimates from Theorem 2.1, (6.7) and (6.9) in (6.6), we arrive at

Claim \#3: There exist a constant $C$ independent of $\varepsilon, R$ and for any $R \in \mathbb{R}_{+}$, $0<\varepsilon<1$ a positive constant $\sigma_{\varepsilon}(R)$ such that

$$
\lim _{\varepsilon \rightarrow 0} \sigma_{\varepsilon}(R)=0
$$

for any fixed $R$, and

$$
\left|I_{\varepsilon}\right| \leq C\left(\frac{m}{R}+\sigma_{\varepsilon}(R)+\max _{\left|x-x^{0}\right| \leq R_{\varepsilon}, 0 \leq t \leq T}\left|\varphi\left(x^{0}, t\right)-\varphi(x, t)\right|\right) .
$$

Proof of Claim \#3: Indeed, as

$$
\begin{array}{r}
\left|\varphi(x, t)-\varphi_{\varepsilon}(x, t)\right| \leq|\varphi(x, t)|\left|\xi_{\varepsilon}(x, R)\right|+\frac{1+\left|\xi_{\varepsilon}(x, R)\right|}{1-\operatorname{ess}_{x^{0}} \sup _{x^{0}}\left|\theta_{\varepsilon}(x, R)\right|} \\
\times\left(\max _{\left|x-x^{0}\right| \leq R_{\varepsilon}}\left|\varphi\left(x^{0}, t\right)-\varphi(x, t)\right|+\operatorname{ess} \sup _{x^{0} \in \partial \Omega}\left|\theta_{\varepsilon}\left(x^{0}, R\right)\right|\right),
\end{array}
$$

the assertion of Claim \# 3 follows easily due to (6.7) and (6.9).

Claim \#4: There exists a constant $C_{m}$ independent of $\varepsilon, R$ and for any $R \in \mathbb{R}_{+}$and $0<\varepsilon<1$ there exists a positive constant $s_{\varepsilon}(R)$ such that

$$
\begin{aligned}
I I_{\varepsilon}= & \int_{\partial \Omega_{T}} f_{B^{\prime}(R)} \omega\left(x^{0}, z^{\prime}, 0\right) d z^{\prime} g\left(x^{0}, t\right) \varphi\left(x^{0}, t\right) d H^{n-1}\left(x^{0}\right) d t \\
& +s_{\varepsilon}(R)+\frac{C_{m}}{R}
\end{aligned}
$$

and

$$
\lim _{\varepsilon \rightarrow 0} s_{\varepsilon}(R)=0
$$

for any fixed $R$. 
Proof of Claim \#4: Note that due to the notation prior to (6.7),

$$
\begin{aligned}
I I_{\varepsilon}=\int_{\mathcal{G}_{T}^{\kappa}} \frac{1}{1+\theta_{\varepsilon}\left(x^{0}, R\right)} & \int_{B^{\prime}(R)} v_{\varepsilon}\left(x^{0}, z^{\prime}, 0, t\right) \sqrt{1+\left|D \gamma\left(x^{0}, \varepsilon \Psi_{\varepsilon}^{\prime}\right)\right|^{2}} \\
& \times J \Psi_{\varepsilon}^{\prime}\left(x^{0}, z^{\prime}-\tau_{\varepsilon}^{\prime}\left(x^{0}\right)\right) d z^{\prime} \varphi\left(x^{0}, t\right) d H^{n-1}\left(x^{0}\right) d t
\end{aligned}
$$

and the assertion follows due to (6.7), (5.3) and Corollary 5.2.

Now we are ready to prove (6.5). Employing Claim \# 3 and Claim \# 4 we can write

$$
\begin{aligned}
&\left|\int_{\partial \Omega_{T}}\left(W_{\varepsilon}\left(x^{0}, t\right)-M\left(x^{0}\right) g\left(x^{0}, t\right)\right) \varphi\left(x^{0}, t\right) d H^{n-1}\left(x^{0}\right) d t\right| \\
& \leq \frac{C}{R}+\mathcal{O}_{\varepsilon}(R)+C \max _{\left|x-x^{0}\right| \leq R_{\varepsilon}, 0 \leq t \leq T}\left|\varphi\left(x^{0}, t\right)-\varphi(x, t)\right| \\
&+\int_{\partial \Omega_{T}}\left|f_{B^{\prime}(R)} \omega\left(x^{0}, z^{\prime}, 0\right) d z^{\prime}-M\left(x^{0}\right) g\left(x^{0}, t\right)\right| \\
&\left|\varphi\left(x^{0}, t\right)\right| d H^{n-1}\left(x^{0}\right) d t
\end{aligned}
$$

where

$$
\lim _{\varepsilon \rightarrow 0} \mathcal{O}_{\varepsilon}(R)=0 \quad \text { for fixed } R .
$$

Finally, we choose $R$ large enough in order to have the first term and the last one on the right hand side of (6.10) less than $\eta / 2$ and then $\varepsilon_{0}$ small enough to have the additional two terms less than $\eta / 2$ for each $0<\varepsilon<\varepsilon_{0}$.

We have so far proved that for any $X \in \mathcal{G}$ there exists a ball $B(X, \iota)$, $\iota>0$ such that for $\mathcal{H}^{n-1}$ a.e. $x \in \partial \Omega \cap B(X, \iota), W_{0}(x, t)=M(x) g(x, t)$. Therefore, we can conclude that this equality holds for $\mathcal{H}^{n-1}$ a.e. $x \in \partial \Omega$ and the theorem follows.

\section{Capacity}

In this section we introduce capacity as a way to find sufficiently general structural conditions on the set $\mathbb{D}$ in order to guarantee the existence of the mean value of $\omega$ for $x_{n}=0$, see (6.4) above. As the dependence of $\omega$ on $x^{0} \in \partial \Omega$ does not play any role in this considerations, let us omit to write it down here. Recall

$$
\begin{aligned}
\Delta \omega=0 & \text { in } \mathbb{R}_{+}^{n}, \\
\omega=0 & \text { on } \mathbb{D}_{0}, \\
\partial_{\nu} \omega=1 & \text { on } \mathbb{N}_{0},
\end{aligned}
$$


$\mathbb{D}_{0} \equiv \mathbb{D} \times\left\{x_{n}=0\right\}$, and till now we have supposed that $\mathbb{D}$ is asymptotically dense in $\mathbb{R}^{n-1}$ (see Definition 3.1). In order to be able to formulate our result, it is convenient to introduce some preliminaries, developed in the theory of almost periodic functions (see e.g. [2], [10], [11]).

Definition 7.1. Given a function $v \in L^{\infty}\left(\mathbb{R}^{n-1}\right)$ and numbers $0<\eta<1$, $0<R_{\eta}<\infty$, a vector $\lambda \in \mathbb{R}^{n-1}$ is said to be a translation vector belonging to $\eta$ if

$$
\sup _{R \geq R_{\eta}} f_{C^{\prime}(R)}\left|v\left(x^{\prime}+\lambda\right)-v\left(x^{\prime}\right)\right| d x^{\prime} \leq \eta
$$

Recall, $C^{\prime}(R) \equiv\left\{y^{\prime} \in \mathbb{R}^{n-1}|| y_{i} \mid<R, i=1,2, \ldots, n-1\right\}$.

A set $E \subset \mathbb{R}^{n-1}$ is said to be $\ell$-relatively dense if there is a number $\ell>0$ such that any cube of side $\ell$ in $\mathbb{R}^{n-1}$ contains at least one point of $E$.

Theorem 7.2. Assume that for any $\eta>0$ correspond numbers $R_{\eta}>0$ and $\ell_{\eta}>0$ such that the set of all translation vectors of $v \in L^{\infty}\left(\mathbb{R}^{n-1}\right)$ belonging to $\eta$ is $\ell_{\eta}-$ relatively dense in $\mathbb{R}^{n-1}$. Then the mean value of $v$ exists, i.e. the limit of

$$
f_{B^{\prime}(R)} v\left(x^{\prime}\right) d x^{\prime} \quad \text { as } R \rightarrow \infty,
$$

exists.

Proof. Let $k \in\{1,2, \ldots\}$ and denote by $C^{\prime}\left(x^{i}, R\right)$ for $x^{i} \in B^{\prime}(k R)$ open disjoint cubes in $\mathbb{R}^{n-1}$, i.e.

$$
C^{\prime}\left(x^{i}, R\right) \equiv\left\{y^{\prime} \in \mathbb{R}^{n-1}|| x_{j}^{i}-y_{j} \mid<R, j=1, \ldots, n-1\right\}
$$

such that

$$
B^{\prime}(k R) \subset C_{k} \equiv \bigcup_{i=1}^{N_{k}} \bar{C}^{\prime}\left(x^{i}, R\right) \subset B^{\prime}((k+2) R) .
$$

As for $A \subset C \subset D$,

$$
\left|f_{A} v-f_{C} v\right| \leq 2\|v\|_{L^{\infty}\left(\mathbb{R}^{n-1}\right)} \frac{\mathcal{L}^{n-1}(D)-\mathcal{L}^{n-1}(A)}{\mathcal{L}^{n-1}(A)},
$$

we have

$$
\left|f_{B^{\prime}(k R)} v\left(x^{\prime}\right) d x^{\prime}-f_{C_{k}} v\left(x^{\prime}\right) d x^{\prime}\right| \leq \frac{c}{k}
$$


with a constant $c \in \mathbb{R}_{+}$independent of $k, R$. Compute now

$$
\begin{aligned}
& \int_{C_{k}} v\left(x^{\prime}\right) d x^{\prime}=\sum_{i=1}^{N_{k}}\left[\int_{C^{\prime}(R)} v\left(y^{\prime}+\lambda^{i}\right) d y^{\prime}+\int_{C^{\prime}\left(x^{i}-\lambda^{i}, R\right) \backslash C^{\prime}(R)} v\left(y^{\prime}+\lambda^{i}\right) d y^{\prime}\right. \\
& \left.-\int_{C^{\prime}(R) \backslash C^{\prime}\left(x^{i}-\lambda^{i}, R\right)} v\left(y^{\prime}+\lambda^{i}\right) d y^{\prime}\right] \\
& =N_{k} \int_{C^{\prime}(R)} v\left(y^{\prime}\right) d y^{\prime}+\sum_{i=1}^{N_{k}} \int_{C^{\prime}(R)}\left|v\left(y^{\prime}+\lambda^{i}\right)-v\left(y^{\prime}\right)\right| d y^{\prime}+R^{n-1} N_{k} \theta_{R} \text {, }
\end{aligned}
$$

where

i.e.

$$
\left|\theta_{R}\right| \leq \frac{c}{R}
$$

$$
f_{C_{k}} v\left(x^{\prime}\right) d x^{\prime}=f_{C^{\prime}(R)} v\left(y^{\prime}\right) d y^{\prime}+\vartheta_{\eta} \eta+\theta_{R},
$$

for some $\left|\vartheta_{\eta}\right| \leq 1$. In summary,

$$
\left|f_{B^{\prime}(k R)} v\left(x^{\prime}\right) d x^{\prime}-f_{B^{\prime}(\ell R)} v\left(x^{\prime}\right) d x^{\prime}\right| \leq \eta+c\left(\frac{1}{R}+\frac{1}{k}+\frac{1}{\ell}\right) .
$$

Thus, for arbitrarily large numbers $X_{j}, j=1,2$ let $k_{j}$ be such that $k_{j} R \leq$ $X_{j}<k_{j} R+1$ and we arrive at

$$
\left|f_{B^{\prime}\left(X_{1}\right)} v\left(x^{\prime}\right) d x^{\prime}-f_{B^{\prime}\left(X_{2}\right)} v\left(x^{\prime}\right) d x^{\prime}\right| \leq c\left(\eta+\frac{1}{R}+\frac{1}{k_{1}}+\frac{1}{k_{2}}\right)
$$

and the assertion of Theorem 7.2. follows.

Our plan is hereafter to find circumstances under which bounded weak solution $\omega$ of Problem (7.1) satisfies hypotheses of Theorem 7.2 for $x_{n}=0$. We propose here the following assumptions. Denote

$$
\mathbb{D}^{\lambda} \equiv \mathbb{D}+\lambda, \quad \mathbb{D}_{0}^{\lambda} \equiv \mathbb{D}^{\lambda} \times\left\{x_{n}=0\right\}
$$

for $\lambda \in \mathbb{R}^{n-1}$, recall $\mathbb{D}_{0} \Delta \mathbb{D}_{0}^{\lambda} \equiv \mathbb{D}_{0} \cup \mathbb{D}_{0}^{\lambda} \backslash \mathbb{D}_{0} \cap \mathbb{D}_{0}^{\lambda}$ and define

$$
\operatorname{Cap}_{L}\left(\mathbb{D}_{0} \triangle \mathbb{D}_{0}^{\lambda}\right) \equiv \sup _{R \geq L} R^{1-n} \inf _{\mathcal{H} \in V(R)} \int_{B_{+}(R)}|\nabla \mathcal{H}|^{2},
$$

where

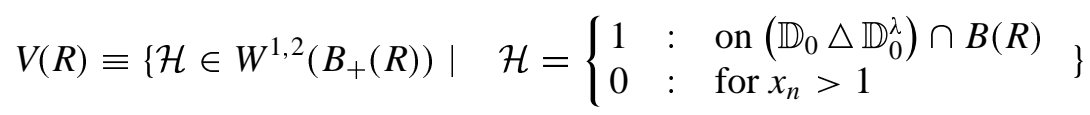

and $L \in \mathbb{R}_{+}, \lambda \in \mathbb{R}^{n-1}$ given. 
Assumption (P). For any $\eta>0$ there exist positive numbers $\ell_{\eta}, L_{\eta}$ and $a$ $\ell_{\eta}-$ relatively dense set $E_{\eta}$ in $\mathbb{R}^{n-1}$ such that

$$
\operatorname{Cap}_{L_{\eta}}\left(\mathbb{D}_{0} \triangle \mathbb{D}_{0}^{\lambda}\right) \leq \eta
$$

for any $\lambda \in E_{\eta}$.

Theorem 7.3. Assume that $\omega$ is a bounded weak solution of Problem (7.1), where the set $\mathbb{D}$ satisfies Assumption $(P)$.

Then the function $\omega\left(x^{\prime}, 0\right)$ for $x^{\prime} \in \mathbb{R}^{n-1}$ satisfies all hypotheses required in Theorem 7.2 above.

Proof. 1. Fix $\lambda \in E_{\eta}$ given in Assumption (P) and define

$$
u(x) \equiv \omega\left(x^{\prime}+\lambda, x_{n}\right)-\omega\left(x^{\prime}, x_{n}\right), \quad u^{D}\left(x^{\prime}\right) \equiv u\left(x^{\prime}, 0\right) .
$$

Then

$$
\begin{aligned}
\Delta u & =0 & & \text { in } \mathbb{R}_{+}^{n}, \\
u & =0 & & \text { on } \mathbb{D}_{0} \cap \mathbb{D}_{0}^{\lambda}, \\
u & =u^{D} & & \text { on } \mathbb{D}_{0} \triangle \mathbb{D}_{0}^{\lambda}, \\
\partial_{\nu} u & =0 & & \text { on } \mathbb{N}_{0} \cap \mathbb{N}_{0}^{\lambda},
\end{aligned}
$$

in the sense that $u \in W_{\text {loc }}^{1,2}\left(\mathbb{R}_{+}^{n}\right), u=u^{D}$ on $\mathbb{D}_{0} \triangle \mathbb{D}_{0}^{\lambda}$ and

$$
\int_{\mathbb{R}_{+}^{n}} \nabla u \nabla \psi=0
$$

for any $\psi \in W_{\text {loc }}^{1,2}\left(\mathbb{R}_{+}^{n}\right), \psi=0$ on $\mathbb{D}_{0} \cup \mathbb{D}_{0}^{\lambda}$, with a compact support in $\mathbb{R}_{+}^{n}$.

2. Take next sufficiently large $R$ and insert

$$
\psi=u \phi_{R}^{2}\left(1-\mathcal{H}_{R}\right)^{2}
$$

as a test function in (7.5), where functions $\mathcal{H}_{R}$ and $\phi_{R}$ are defined as follows: $\mathcal{H}_{R}$ is the minimizer of Dirichlet's integral

$$
J(\mathcal{H})=\int_{B_{+}(3 R)}|\nabla \mathcal{H}|^{2}
$$

on $V(3 R)$ (see (7.3) above), i.e.

$$
\begin{aligned}
\Delta \mathcal{H}_{R}=0 & \text { in } U_{3 R} \equiv B_{+}(3 R) \cap\left\{x \mid 0<x_{n}<1\right\}, \\
\mathcal{H}_{R}=0 & \text { on } B_{+}(3 R) \backslash U_{3 R}, \\
\mathcal{H}_{R}=1 & \text { on }\left(\mathbb{D}_{0} \triangle \mathbb{D}_{0}^{\lambda}\right) \cap B(3 R), \\
\partial_{\nu} \mathcal{H}_{R}=0 & \text { on } S_{3 R},
\end{aligned}
$$


where

$$
S_{3 R} \equiv \partial U_{3 R} \backslash\left(\left\{x \in \bar{B}_{+}(3 R) \mid x_{n}=1\right\} \cup\left(\mathbb{D}_{0} \cap \mathbb{D}_{0}^{\lambda}\right)\right)
$$

and

$$
\begin{aligned}
\Delta \phi_{R}=0 & \text { in } B_{+}(3 R), \\
\phi_{R}=0 & \text { on } \partial B_{+}(3 R) \cap \mathbb{R}_{+}^{n}, \\
\phi_{R}=1 & \text { on } B(2 R) \cap\left\{x \mid x_{n}=0\right\}, \\
\partial_{\nu} \phi_{R}=0 & \text { on }(B(3 R) \backslash B(2 R)) \cap\left\{x \mid x_{n}=0\right\} .
\end{aligned}
$$

Thus,

$$
\begin{gathered}
\int_{B_{+}(3 R)}|\nabla u|^{2}\left(1-\mathcal{H}_{R}\right)^{2} \phi_{R}^{2} \\
\leq C_{1}\left(\int_{B^{+}(3 R)}\left|\nabla \mathcal{H}_{R}\right|^{2}|u|^{2} \phi_{R}^{2}+\int_{B^{+}(3 R)}\left|\nabla \phi_{R}\right|^{2}\left(1-\mathcal{H}_{R}\right)^{2}|u|^{2}\right) .
\end{gathered}
$$

Since,

$$
\int_{B_{+}(3 R)}\left|\nabla \phi_{R}\right|^{2} \leq C_{2} R^{n-2},
$$

$0 \leq \phi_{R}, \mathcal{H}_{R} \leq 1$ on $B_{+}(3 R), 0<v \leq \phi_{R}$ on $B_{+}(2 R)$ and $u \in L^{\infty}\left(\mathbb{R}_{+}^{n}\right)$, the inequality above implies

$$
\int_{B_{+}(2 R)}\left|\nabla\left(u\left(1-\mathcal{H}_{R}\right)\right)\right|^{2} \leq C_{3}\left(R^{n-2}+\int_{B_{+}(3 R)}\left|\nabla \mathcal{H}_{R}\right|^{2}\right) .
$$

Note that the facts that $\mathbb{D}$ is asymptotically dense in $\mathbb{R}^{n-1}, u\left(1-\mathcal{H}_{R}\right)=0$ on $\mathbb{D}_{0} \cup \mathbb{D}_{0}^{\lambda}$ and (7.6) yield

$$
\int_{B_{+}(2 R) \cap\left\{x \mid x_{n}=1\right\}}|u|^{2} \leq C_{4}\left(R^{n-2}+\int_{B_{+}(3 R)}\left|\nabla \mathcal{H}_{R}\right|^{2}\right) .
$$

3. Assume now that $u_{h}$ is a solution of

$$
\begin{aligned}
\Delta u_{h} & =0 & & \text { in } U_{2 R}, \\
u_{h} & =|u| & & \text { on } B_{+}(2 R) \cap\left\{x \mid x_{n}=1\right\}, \\
u_{h} & =0 & & \text { on }\left(\mathbb{D}_{0} \cup \mathbb{D}_{0}^{\lambda}\right) \cap B(2 R), \\
\partial_{\nu} u_{h} & =0 & & \text { on } S_{2 R},
\end{aligned}
$$

( $S_{2 R}$ is the rest part of $\partial U_{2 R}$ ). Note that $u_{h}$ is the minimizer of the Dirichlet's integral $J(w)=\int_{U_{2 R}}|\nabla w|^{2}$ over the set

$$
X \equiv\left\{w \in W^{1,2}\left(U_{2 R}\right)|\quad w|_{x_{n}=1}=|u| \text { and } \quad w=0 \quad \text { on } \quad \mathbb{D}_{0} \cup \mathbb{D}_{0}^{\lambda}\right\} .
$$


As $|u|\left(1-\mathcal{H}_{R}\right) \in X,(7.6)$ yields

$$
\int_{U_{2 R}}\left|\nabla u_{h}\right|^{2} \leq C\left(R^{n-2}+\int_{B_{+}(3 R)}\left|\nabla \mathcal{H}_{R}\right|^{2}\right) .
$$

4. Claim \#1: Let $M \equiv\|\omega\|_{L^{\infty}\left(\mathbb{R}_{+}^{n}\right)}$, then

$$
|u| \leq u_{h}+M \mathcal{H}_{R}+2 M \varphi_{R}
$$

on $U_{2 R}$, where

$$
\begin{aligned}
\Delta \varphi_{R}=0 & \text { in } B_{+}(2 R), \\
\varphi_{R}=0 & \text { on }\left(\mathbb{D}_{0} \cup \mathbb{D}_{0}^{\lambda}\right) \cap B(2 R), \\
\varphi_{R}=1 & \text { if }|x|=2 R, \\
\partial_{\nu} \varphi_{R}=0 & \text { on } S_{2 R},
\end{aligned}
$$

where $S_{2 R}$ denotes the rest of $\partial B_{+}(2 R)$.

Proof of Claim \#1. Note first that $|u|$ is a subsolution of the problem

$$
\begin{aligned}
\Delta v & =0 & & \text { in } U_{2 R}, \\
v & =0 & & \text { on }\left(\mathbb{D}_{0} \cap \mathbb{D}_{0}^{\lambda}\right) \cap B(2 R), \\
v & =\left|u^{D}\right| & & \text { on }\left(\mathbb{D}_{0} \Delta \mathbb{D}_{0}^{\lambda}\right) \cap B(2 R), \\
v & =|u| & & \text { on } B_{+}(2 R) \cap\left\{x \mid x_{n}=1 \text { or }|x|=2 R\right\}, \\
\partial_{\nu} v & =0 & & \text { on }\left(\mathbb{N}_{0} \cap \mathbb{N}_{0}^{\lambda}\right) \cap B(2 R),
\end{aligned}
$$

i.e.

$$
\int_{U_{2 R}} \nabla|u| \nabla \psi d x \leq 0
$$

for any $\psi \in W^{1,2}\left(U_{2 R}\right), \quad \psi \geq 0$ and $\psi=0$ on $\mathbb{D}_{0} \cap \mathbb{D}_{0}^{\lambda}$ and on $\left\{x \mid x_{n}=1\right.$ or $|x|=2 R\} \cap \bar{U}_{2 R}$.

The function $V=u_{h}+M \mathcal{H}_{R}+2 M \varphi_{R}$ is, however, a supersolution of Problem (7.9), i.e.

$$
|u| \leq V
$$

on $U_{2 R}$ and the assertion follows.

5. Finally, Claim \#1 yields

$$
\begin{aligned}
\int_{B^{\prime}(R)} & \left|u\left(x^{\prime}, 0\right)\right|^{2} d x^{\prime} \\
& \leq C_{5}\left(\left\|u_{h}\right\|_{W^{1,2}\left(U_{2 R}\right)}^{2}+\left\|\mathcal{H}_{R}\right\|_{W^{1,2}\left(U_{2 R}\right)}^{2}+\int_{B^{\prime}(R)}\left|\varphi_{R}\left(x^{\prime}, 0\right)\right|^{2} d x^{\prime}\right) \\
& \leq C_{6}\left(R^{n-2}+\int_{B_{+}(3 R)}\left|\nabla \mathcal{H}_{R}\right|^{2}+\int_{B^{\prime}(R)}\left|\varphi_{R}\left(x^{\prime}, 0\right)\right|^{2} d x^{\prime}\right),
\end{aligned}
$$


where (7.7), (7.8) have been taking account. It remains to estimate the last integral on the right hand side of (7.10). Here we apply arguments of the proof of Theorem 3.3 and we can conclude that

$$
\left|\varphi_{R}\right| \leq C / R
$$

on $B^{\prime}(R) \times\left\{x \mid 0 \leq x_{n} \leq 1\right\}$ for some positive constants $C$ independent of $R$. Hence,

$$
f_{B^{\prime}(R)}\left|\omega\left(x^{\prime}+\lambda, 0\right)-\omega\left(x^{\prime}, 0\right)\right| d x^{\prime} \leq C_{7}\left(\frac{1}{R}+f_{B_{+}(3 R)}\left|\nabla \mathcal{H}_{R}\right|^{2}\right)^{1 / 2}
$$

and this completes the proof of the theorem.

\section{Applications}

In this section we identify conditions ensuring the existence of a nontrivial structure of the set $\mathbb{D}$ satisfying Assumption $(\mathrm{P})$ of the previous section.

By the trivial structure of the set $\mathbb{D}$ we mean its periodic structure. To be more precise, let $F: \mathbb{R}^{n-1} \rightarrow\{0,1\}$ be a periodic function in each of its variable, say, with period 1 and let there are $y^{\prime} \in \mathbb{R}^{n-1}$ and $\iota>0$ such that $B\left(y^{\prime}, \iota\right) \subset\left\{x^{\prime} \in \mathbb{R}^{n-1} \mid F\left(x^{\prime}\right)=1\right\}$. In addition to, let $\boldsymbol{A} \in L\left(\mathbb{R}^{n-1}, \mathbb{R}^{n-1}\right)$ be a regular matrix, i.e. $\operatorname{det} \boldsymbol{A} \neq 0$. If we define

$$
\chi\left(x^{\prime}\right) \equiv F\left(\boldsymbol{A} x^{\prime}\right) \quad \text { for } \quad x^{\prime} \in \mathbb{R}^{n-1}
$$

and

$$
\mathbb{D} \equiv\left\{x^{\prime} \in \mathbb{R}^{n-1} \mid \quad \chi\left(x^{\prime}\right)=1\right\},
$$

then $\mathbb{D}$ satisfies Assumption (P) trivially and it is asymptotically dense as well.

Definition 8.1. We shall call a function $\chi: \mathbb{R}^{n-1} \rightarrow\{0,1\}$ quasiperiodic if for any $\eta>0$ there exists a continuous quasiperiodic function $\varphi_{\eta}$ such that the set

$$
\Lambda_{\eta} \equiv\left\{x^{\prime} \in \mathbb{R}^{n-1}|\quad| \chi\left(x^{\prime}\right)-\varphi_{\eta}\left(x^{\prime}\right) \mid>0\right\}
$$

has a small capacity in the following sense: Let $\mathcal{H}_{\eta}$ satisfy

$$
\begin{aligned}
\Delta \mathcal{H}_{\eta}=0 & & \text { in } \mathbb{R}^{n-1} \times(0,1), \\
\mathcal{H}_{\eta}\left(x^{\prime}, 0\right)=1 & & \text { for } x^{\prime} \in \Lambda_{\eta}, \\
\mathcal{H}_{\eta}\left(x^{\prime}, 1\right)=0 & & \text { for } x^{\prime} \in \mathbb{R}^{n-1}, \\
\partial_{\nu} \mathcal{H}_{\eta}\left(x^{\prime}, 0\right)=0 & & \text { for } x^{\prime} \in \mathbb{R}^{n-1} \backslash \Lambda_{\eta},
\end{aligned}
$$


then

$$
\mathcal{C}\left(\Lambda_{\eta}\right) \equiv \sup _{\hat{x} \in \mathbb{R}^{n-1} \times\left\{x_{n}=0\right\}} \sup _{R \geq 1} f_{B(\hat{x}, R) \cap\left\{x \mid 0<x_{n}<1\right\}}\left|\nabla \mathcal{H}_{\varepsilon}\right|^{2} d x \leq \eta .
$$

Proposition 8.2. Let $\chi: \mathbb{R}^{n-1} \rightarrow\{0,1\}$ be a quasiperiodic function and assume that there are $y^{\prime} \in \mathbb{R}^{n-1}$ and $\iota>0$ such that

$$
B\left(y^{\prime}, \iota\right) \subset \mathbb{D} \equiv\left\{x^{\prime} \in \mathbb{R}^{n-1} \mid \chi\left(x^{\prime}\right)=1\right\} .
$$

Then the set $\mathbb{D}$ satisfies Assumption $(P)$ (see (7.4) above) and it is the asymptotically dense set in the sense of Definition 3.1.

Proof. Note first that by a continuous quasiperiodic function in $\mathbb{R}^{n-1}$ we mean a function $\varphi \in C\left(\mathbb{R}^{n-1}\right)$ such that

$$
\varphi\left(y^{\prime}\right) \equiv f\left(\boldsymbol{B} y^{\prime}\right),
$$

where $f \in C\left(\mathbb{R}^{k}\right), k \geq 1$ is periodic in each of its variable with period 1 and $\boldsymbol{B} \in L\left(\mathbb{R}^{n-1}, \mathbb{R}^{k}\right)$. Moreover, without loss of generality one may assume that

$$
\boldsymbol{B} \cdot \mathbb{R}^{n-1}+\mathbb{Z}^{k}
$$

is dense in $\mathbb{R}^{k}$ (see [12]).

Our aim is to show that for any $\eta>0$ there exist positive numbers $\ell_{\eta}, L$ and $\ell_{\eta}$ - relative dense set $E_{\eta} \subset \mathbb{R}^{n-1}$ such that

$$
\operatorname{Cap}_{L}\left(\mathbb{D}_{0} \triangle \mathbb{D}_{0}^{\lambda}\right) \leq \eta
$$

for any $\lambda \in E_{\eta}$.

1. As $\chi$ is supposed to be quasiperiodic, for any $\eta>0$ there exists a continuous quasiperiodic function $\varphi_{\eta}$ such that

$$
\mathcal{C}\left(\Lambda_{\eta}\right) \leq \eta
$$

Fix $\eta>0$. It follows from Kronecker-Weyl's Theorem (see e.g. [10, 11], [12]) that for any $\delta>0$ there exists $\ell_{\delta}$ and a $\ell_{\delta}-$ relative dense set $E_{\delta}$ in $\mathbb{R}^{n-1}$ such that for any $\lambda \in E_{\delta}$,

$$
\varphi_{\eta}\left(x^{\prime}+\lambda\right)-\varphi_{\eta}\left(x^{\prime}\right)=\varphi_{\eta}\left(x^{\prime}+\lambda_{\delta}\left(x^{\prime}\right)\right)-\varphi_{\eta}\left(x^{\prime}\right)
$$

and

$$
\left|\lambda_{\delta}\left(x^{\prime}\right)\right|<\delta
$$


$x^{\prime} \in \mathbb{R}^{n-1}$ being arbitrary. As $\varphi_{\eta}$ is uniformly continuous on $\mathbb{R}^{n-1}$ (see (8.3)), (8.4) yields that $\left|\varphi_{\eta}\left(x^{\prime}+\lambda\right)-\varphi_{\eta}\left(x^{\prime}\right)\right|<1$, say,

$$
\max _{x^{\prime} \in \mathbb{R}^{n-1}}\left|\varphi_{\eta}\left(x^{\prime}+\lambda\right)-\varphi_{\eta}\left(x^{\prime}\right)\right| \leq 1 / 2
$$

if $\delta$ is choosen sufficiently small.

2. If $\lambda \in E_{\delta}$,

$$
\begin{array}{r}
\left|\chi\left(x^{\prime}+\lambda\right)-\chi\left(x^{\prime}\right)\right| \leq\left|\chi\left(x^{\prime}+\lambda\right)-\varphi_{\eta}\left(x^{\prime}+\lambda\right)\right|+1 / 2 \\
+\left|\varphi_{\eta}\left(x^{\prime}\right)-\chi\left(x^{\prime}\right)\right|,
\end{array}
$$

wherein the left hand side can attain only two values, zero and one. If $\left|\chi\left(x^{\prime}+\lambda\right)-\varphi_{\eta}\left(x^{\prime}+\lambda\right)\right|=0$ and at the same time $\left|\varphi_{\eta}\left(x^{\prime}\right)-\chi\left(x^{\prime}\right)\right|=0$, then also $\left|\chi\left(x^{\prime}+\lambda\right)-\chi\left(x^{\prime}\right)\right|=0$.

Thus, $\left|\chi\left(x^{\prime}+\lambda\right)-\chi\left(x^{\prime}\right)\right|$ could equal one only if $x^{\prime} \in \Lambda_{\eta}$ or $x^{\prime}+\lambda \in \Lambda_{\eta}$. This yields

$$
\mathbb{D}_{0} \triangle \mathbb{D}_{0}^{\lambda} \subset \Lambda_{\eta}
$$

hence

$$
\operatorname{Cap}_{1}\left(\mathbb{D}_{0} \Delta \mathbb{D}_{0}^{\lambda}\right) \leq \mathcal{C}\left(\Lambda_{\eta}\right) \leq \eta
$$

for any $\lambda \in E_{\eta} \equiv E_{\delta}$. This proves our statement.

\section{References}

1. H.W. Alt, S. Luckhaus: Quasilinear elliptic-parabolic differential equations. Math. Z. 183, 311-341 (1983)

2. A.S. Besicovitch: Almost Periodic Functions. Dover Publications, Inc., 1954

3. A. Bourgeat, S. Luckhaus, A. Mikelic: Convergence of the homogenization process for a double-porosity model of immiscible two-phase flow. SIAM. J. Math. Anal. 27(6), 1520-1543 (1996)

4. L.C. Evans: Partial Differential Equations. American Mathematical Society, 1998

5. L.C. Evans, R.F. Gariepy: Measure Theory and Fine Properties of Functions. CRC Press, 1992

6. J. Filo, S. Luckhaus: Asymptotic expansion for a periodic boundary condition. J. Diff. Equations 120, 133-173 (1995)

7. D. Gilbarg, N.S. Trudinger: Elliptic Partial Differential Equations of Second Order. Berlin, Heidelberg, New York: Springer 1983

8. V.V. Jikov, S.M. Kozlov, O.A. Olejnik: Homogenization of Differential Operators and Integral Functionals. Berlin, Heidelberg, New York: Springer 1994

9. O.A. Ladyzenskaja, V.A. Solonnikov, N.N. Uralceva: Linear and quasilinear equations of parabolic type. Translations of Mathematical Monographs, Vol. 23, Providence, Rhode Island 1968

10. B.M. Levitan: Almost-Periodic Functions. Moscow, 1953

11. B.M. Levitan, V.V. Zhikov: Almost Periodic Functions and Differential Equations. Cambridge: Cambridge University Press 1982

12. H. Weyl: Uber die Gleichverteilung von Zahlen mod. Eins. Math. Ann. 77, 313-352 (1916) 